\title{
Extended Linear and Nonlinear Lorentz Transformations and Superluminality
}

\author{
Dara Faroughy \\ Department of Sciences, Pima College, West Campus, Tucson, AZ 85709, USA \\ Correspondence should be addressed to Dara Faroughy; darifaro@yahoo.com
}

Received 8 November 2012; Revised 1 March 2013; Accepted 7 March 2013

Academic Editor: S. H. Dong

Copyright (C) 2013 Dara Faroughy. This is an open access article distributed under the Creative Commons Attribution License, which permits unrestricted use, distribution, and reproduction in any medium, provided the original work is properly cited.

\begin{abstract}
Two broad scenarios for extended linear Lorentz transformations (ELTs) are modeled in Section 2 for mixing subluminal and superluminal sectors resulting in standard or deformed energy-momentum dispersions. The first scenario is elucidated in the context of four diverse realizations of a continuous function $f(v)$, with $0 \leq f(v) \leq 1$ and $f(0)=f(c)=1$, which is fitted in the ELT. What goes in the making of the ELT in this scenario is not the boost speed $v$, as ascertained by two inertial observers in uniform relative motion (URM), but $v \times f(v)$. The second scenario infers the preexistence of two rest-mass-dependent superluminal speeds whereby the ELTs are finite at the light speed $c$. Particle energies are evaluated in this scenario at $c$ for several particles, including the neutrinos, and are auspiciously found to be below the GKZ energy cutoff and in compliance with a host of worldwide ultrahigh energy cosmic ray data. Section 3 presents two broad scenarios involving a number of novel nonlinear LTs (NLTs) featuring small Lorentz invariance violations (LIVs), as well as resurrecting the notion of simultaneity for limited spacetime events as perceived by two observers in URM. These inquiries corroborate that NLTs could be potent tools for investigating LIVs past the customary LTs.
\end{abstract}

\section{Introduction}

The standard theory of special relativity (SR) is a simple framework, relying on a set of well establish postulates, for mixing and studying the space and time coordinate transformations between systems of inertial frames (IFs) in relative uniform motion. It takes into account the universality of the light speed signals, as the maximum speed attainable in nature, in all IFs regardless of the motion of the light emitting sources, or observers measuring such signals. The mathematical tools in SR for connecting flat spacetime transformations $(x, t) \leftrightarrow\left(x^{\prime}, t^{\prime}\right)$ between any two IFs $K(x, t)$ and $K^{\prime}\left(x^{\prime}, t^{\prime}\right)$, in uniform relative (and subluminal) motion, are the Lorentz transformations (LT). The textbook LT are the mathematical embodiment of the SR postulates and as such are forcibly both invertible and linear sets of equations. Consequently, by design, the LT could neither allow nor are equipped to deal with faster than light speeds or, independently, allow for any likely nonlinear spacetime connections between the IFs. The embodiments of the latter two topics in an extended version(s) of LT are the main themes of interest in this work. In principle, one can study these themes independently of each other, or assimilate them together. Sections 2 and 3 deal with such possibilities. This paper, however, does not cover the possibility of superluminal phenomena for the case of massless particles, like photons, or the emergence of such phenomena due to any particle coupling to gravity.

The contentious notion of superluminality (SL), alleged to invalidate the relativity principle, and perhaps the notion of locality in quantum mechanics, and led also to undesirable causal paradoxes associated with the time travel, has been a subject of debates in physics for a long time. The term SL refers to any motion or signal traveling faster than the universal light speed $c$. With the Lorentz symmetry so well established experimentally, violation of this symmetry due to any existing SL anomaly has to be very small, at least at the level of energies accessible today. The solely classical SL models, discussed in the first half of this paper in Section 2, reveal this verity analytically, while also making finite value prediction for all relativistic parameters, such as energy, at the exact light speed. To provide an early example of SL anomaly prediction, we refer to our second scenario discussed in Section 2 (see (17a), (17b), and (17c)). In this scenario, any presently known particle of rest mass $m_{o}$ can reach the Planck 
energy $\approx 1.22 \times 10^{19} \mathrm{GeV}$ at the superluminal speed $c^{*} \approx$ $c\left[1-1 / 2\left(m_{o} / M\right)^{2}\right]$, where $M$ is taken as the Planck mass. Thus, the SL anomaly defined as $\Delta=c^{*} / c-1$ can be easily computed for such particles: $\Delta \approx 1 / 2\left(m_{o} / M\right)^{2}$. For example, for a proton and a neutrino $(\nu)$ of rest mass $\approx 0.01 \mathrm{eV}$, we find, respectively, $\Delta_{P} \approx 3 \times 10^{-39}$ and $\Delta_{v} \approx 3.3 \times 10^{-61}$. As noted, these superluminal anomalies are hopelessly too small, even at the Planck energy, to be detected via all known techniques today, or in the foreseeable future for this matter; in some way the challenge is comparable to detecting the stringy nature of the SM particles by today's experimental capabilities. Yet, things change drastically if in the future particles of much higher rest masses are discovered. For example, for a GUT scale particle of rest mass $\approx 5.5 \times 10^{13} \mathrm{GeV}$, and detected at Planck energy, the SL anomaly amplifies to $10^{-8}$, which may be detectable in, some to be found, ultra-relativistic cosmic ray scenarios. In short, the assumption in Section 2 is that massive elementary particles are endowed with maximal (and rest mass dependent) superluminal speeds that, in contrast to $\mathrm{SR}$, are no longer the light speed, albeit extremely close to $c$.

In view of the dismal numerical values presented earlier for the SL anomalies, one is encouraged to challenge the common belief that two inertial observers receiving a superluminal signal, made of massive particles, could not agree verifiably on whether they received it before or after it was emitted, and such ambiguity is of course absent for luminal signals traveling on the light-cone. Under such exceedingly small SL anomaly, any suspected disagreement between the two observers can only be of semantic value. Because of leaving quantum mechanical subtleties aside, such type disagreements could not be authenticated by any net observational effect due to extremely small SL for them to argue about while communicating via known massive particle exchanges.

The study of Lorentz symmetry breaking is not limited to just SL, as it can, and often does, occur in pertinent theories, even at subluminal speeds, including a number of possible causes that are not the topics of interest to discuss in this paper. For the purpose of this work, however, another suitable cause for breaking the Lorentz symmetry, beside SL, may be traced to the likelihood that the "effective" Lorentz transformations (LT), underlying the theory of special relativity and the causality principle in flat space, may be inherently nonlinear in texture in the classical domains due to an inherent impossibility in probing small regions of spacetime by means of quantum proxies. In such scenarios, the issue of whether nonlinear LT (NLT) ought to include only subluminal speeds or admit also SL is marginalized and only of secondary import. This narrative is explored in some detail in the context of NLT in the second half of this paper described in Section 3.

At present, there is no common consensus among physicists on whether SL truly exists, or should even exist, in nature, nor there is a consensus on whether SL constitutes a valuable or a hindering feature for making progress in theories like the Standard Model (SM), and beyond, supersymmetry and so on. Ultimately, it is up to high precision experiments of the future, designed to probe SL, to convince scientists of its existence or obsolescence. But if SL is detected unmistakably in, for example, the time-of-flight measurements of certain particles like electrons, protons, or neutrinos (which we doubt), then it ought to leave an observable footprint, however tiny, in scores of high precision quantum measurements in, for example, atomic physics and in a host of measured elementary particle scattering crosssections or, in particular, in the observation of the ultrarelativistic cosmic ray energies that we address in Section 2.

Unfortunately, the overall theoretical effect of SL (or the NLT for this matter) which ought to be of import in areas of relativistic QM and QFT, string theory and quantum gravity, cosmology, and so forth, is not well developed and reliable enough (besides some speculative conjectured made by a number of authors in the past) to serve as a working tool for researchers to make precise predictions on how certain SL can influence the spectrum of this or that atom, or affect particle interactions, and so forth, or said differently, to help researchers to dissect the exact impact SL may have on the Feynman diagrammatic rules and his integrals to facilitate the computation of the normally Lorentz invariant, amplitudes, phase space, and the kinematic factors needed to predict superluminally corrected cross-sections of a kind. Since in reality elementary particles are endowed with spin, at present there is no known and universally agreed upon (effective) field theoretical Lagrangian formulation for massive interacting fermions or bosons capable of describing Lorentz breaking effects due to superluminality (in contrast to the more common, subluminal, Lorentz symmetry breaking manifesting either in the Lagrangian and the field equations of motion, or only in the field solution VEV values forcibly acquiring a directionality, and thus breaking the Lorentz symmetry).

Consequently, in this paper, and out of necessity, we will deliberately stay away from issues that necessitate the direct intervention of relativistic quantum mechanics, or QFT of a sort. But to be more specific, we should add that there will be a bit of "quantumness" in both sections in form of Planck mass, or the reduced Planck constant, to evaluate the command of our extended LT. The solely quantum issues are undoubtedly beyond the scope of this preliminary work. Nevertheless, it remains true that a complete effect of SL or NLT in the arena of microphysics cannot truthfully be evaluated in processes dealing with quantum particles without involving the full might of the quantum physics machinery. After all, the nature of spacetime and matter at the fundamental level is alleged to be quantum mechanical in texture (quantum spacetime). And the simplistic geometric spacetime tools to standardize causality, such as the light-cone, may undergo modifications at very high probing energies, and in the presence of gravity in the quantum regimes due to the textbook phenomenon of gravitational Birefringence where one photon helicity can travel subluminally and the other superluminally as the result of photon coupling to gravity.

The problem we are facing at this writing is really twofold, insofar as SL or the NLT is concerned. One is once we venture beyond the well-defined structure of the LT, we have to face the fact that neither of these two topics can be formulated in a unique way, meaning that both suffer from a degree of (design) arbitrariness from the onset. In the case of NLT, especially the ones we find most promising and will cover 
in Section 3, finding the inverse transformations algebraically (by simply changing $v \rightarrow-v$ as in LT) could be daunting, if not impossible. Even if this was possible, the inverse transformations often do not preserve the original forms to facilitate the search for the corresponding transformation group structure, ideally isomorphic to the homogeneous Lorentz group, and the spacetime invariants that goes with such NLT. Thus, whether a group structure, and, hence, the induced flat spacetime global geometry, is possible for the NLT of Section 3 remains in doubt. Our priority, though, is well set in the following sense. We wish to keep our effective NLT models at any cost, even if it means a formal group for such transformations may not exist in small regions of spacetime. The good news, though, is that all the proposed forms of NLT converge to standard LT in the limit of infinitesimal nonlinearities, and in this sense the usual group structure and causality characterizing SR are recovered. In Section 3, we will not indulge in group theoretic discussions related to out NLT. Evidently, at the end of the day, the main thing is the balance between how much is gained minus how much is lost in any endeavor. Judging from the amount of innovative materials discussed in Section 3, we believe that the balance is positive. In Section 3, we force the Minkowski geometry on at least one of the two inertial frames $K$ or $K^{\prime}$ to study the effects of the NLT on the other frame.

The second problem to face is even if we had a unique way of formulating the two topics, which we do not, we would still have to confront the issue of how exactly to fit them into the coherent framework of $\mathrm{QM}$, or to be more inclusive into the still unsettled formalism of quantum gravity, where global Lorentz invariance is surely lost, or even more naively, how to incorporate them in the framework of classical GR in small spacetime regions that are nearly locally flat. It so happens that even at the classical level, there is already host of thorny subtleties to overcome; so, we need not invite more subtleties of quantum origin at this juncture. However, it is obvious that by limiting ourselves to only the idealized and classical world of SR, a world deprived of any gravity, we are also depleting ourselves in the number of quantities we can work with. In other words, our working tools, besides the extended LT, are essentially limited to predicting the extended energy and momentum dispersions, time-of-flight or observed particle speeds, and the likes. In this work, though, we will see how by simply allowing SR to include tiny SL or nonlinearities in the fabric of its spacetime transformations we can venture far enough with only those aforesaid limited set of tools. And, moreover, during the course of exploration, propose a host of novel structures, especially in the NLT case, and then go on exploring many, otherwise unknown, potentialities not imaginable in SR. And as a final bonus, we can do all that while making also certain concrete predictions along the way.

At present, all available experimental data on the neutrino SL are consistent with the neutrinos moving at the light speed; yet, the quality of the data is such that it can neither confirm nor refute any prospective tiny SL, and even much more so at the level of numbers we provided earlier. On experimental grounds, though, what is known to date is the likelihood of discovering significant SL anomaly for known massive particles to be assuredly ruled out at the $\mathrm{TeV}$ probing energy scales like the LHC. Yet, as stressed, tiny SL cannot be discarded at this writing due to the significant uncertainties existing in the host of data readily available from diverse experiments performed on neutrinos and antineutrinos having energies typically in the range $\mathrm{MeV}-\mathrm{GeV}$. In the first half of this paper we capitalize on the authentic existence of tiny SL as an ineluctable facet of nature for all massive particles, and not just the neutrinos. Hence, incorporating SL into relativistic physics becomes a first priority, with the aspiration of deriving finite energy and momentum values for massive particles reaching the exact speed of light. The route pursued here for achieving this goal is to go for an extension of orthodox special relativity by purposely targeting the spacetime Lorentz transformations (LT) therein, and then seek diverse superluminal extension of them.

In summary, the focus in this work is the linear and the nonlinear extensions of the familiar Lorentz transformations which pertain to at least two inertial frames (IFs) moving at uniform relative velocity in the flat preexisting Minkowski space setting. Textbook SR asserts the universality of the light speed in all IF; it abolishes the notion of absolute simultaneity (resulting from the orthodox LT and clock synchronization) and leaves no room for possible superluminal motion for massive particles and rigid IF. Section 2 is devoted to the design of a number of extended linear LT grouped into two broad and distinct versions and incorporating superluminality. As seen in Section 2, by using the extended LT as formulated in the second version, which includes SL, we are able to predict the maximum possible subluminal energies for a host of particles moving at the exact speed of light $c$. As it turns out, the latter predictions are not inconsistent with the measured upper energies observed for a number of ultrarelativistic cosmic ray particles.

Section 3, on the other hand, is devoted entirely to the exploration of diverse and often complex nonlinear extensions of the LT (NLT) operating between two IFs in uniform relative motion in $M^{4}$. This section is satiated with technical novelties never conceivable in the standard SR. In this section, we also provide in some detail the various (model dependent) corrections to the orthodox LT of SR for cases when the nonlinearities are small. Interestingly, for few of our models, it may be possible to resurrect the long departed notion of simultaneity and show analytically the existence of instantaneity in two uniformly moving IFs $K$ and $K^{\prime}$ for only a rather constraint region of spacetime events in either frame. The latter finding can have a yawning connotation for a single quantum particle instantaneous correlation (only if its spacetime trajectory includes such constraint events), or in Bell's jargon connectedness, with two distant experimentally indistinguishable inertial observers living in $K$ and $K^{\prime}$. Evidently, we gather that simultaneity may be possible without the need of invoking superluminality. As for SL inclusion in the NLT context, although it can be incorporated in most NLT proposals formulated in Section 3, in order to keep this paper reasonably short, we will consider only subluminal speeds. Even though there is no unique recipe for designing the NLT, and the corresponding extended Poincaré algebra to go with it, and this obviously creates a challenge for making 
attempts to design suitable invariant Lagrangians, that in all likelihood do not exist, even for the simplest case of massive scalar fields, the effort is still worth pursuing. This effort can be undertaken by first focusing on a class of promising NLT whereby the nonlinearities, which normally epitomize a breaking of the standard Lorentz invariance, are small enough, compared to the inbuilt length scale in the nonlinear expressions, to be treated as diminutive perturbation in the spacetime transformations. On the whole, and based on the experience gained in Section 3, we can assert with confidence that with the help of a mild dose of imagination a great deal of technical goodies and potentialities can come out of designing and studying novel forms of NLT, some of which are already worked out in this paper (see also the examples provided later).

An example in designing a class of subluminal NLT that we will explore later is to treat the small nonlinearities in the NLT as propagating energy less abstract waves of a kind satisfying the wave equation in $M^{4}$ (analogous to harmonic coordinates in GR) and then see what ensues (for more see Section 3). (Interestingly, in one scenario belonging to this class we find signs of superluminal wave propagation.) Another NLT scenario, also illustrating a degree of novelty at the prequantum level can go like this. Assert the effective spacetime coordinate transformations between $K$ and $K^{\prime}$ IF, in constant relative motion, for all undetected classical point particles are nonlinear in Nature. Such NLT relate, as usual, measurements in the two different IF. Next, go on devising NLT that can be broken down into the sum of two separable parts, the usual linear LT part and the nonlinear part. Next, affirm that for all particles that are eventually detected (i.e., after the wavefunction collapse, if particles were to be treated as quantum objects, which is an added requirement at this stage), the information derived by such observations must be in concordance with only what is expected from SR, hence, in compliance with the usual Lorentz symmetry. Such a scenario forces the detected particles to appear to the observer making the continuous measurement in say $K^{\prime}$ or $K$ as masses moving along only certain constraint locations in $K$ or $K^{\prime}$ that vanish the nonlinear terms in the NLT, clearly if such locations existed in the boost direction they would, in turn, also affect the linear LT part. In case the nonlinear terms are modeled as a superposition of real periodic functions of a kind (complex spacetime coordinates in $M^{4}$ are not considered here), then from this rationalistic mechanics view instantaneous observation entails observing instantaneous quantized particle trajectories and energies. Now, since an unobserved particle keeps on evolving in time, the unobservable nonlinear terms in the NLT can be viewed as an epistemic uncertainty in locating an unobserved particle in spacetime by the observer living in, for example, $K^{\prime}$.

Anyway, what we have here is a crude example of how observable and time evolving quantized spacetime trajectories and energies in $K$ could stem from the vanishing of the nonlinear terms in the NLT (which in turn were forced upon by the preservation of the exact Lorentz symmetry while the measurement lasts) that ultimately convert to discrete data upon a continuous measurement in $K^{\prime}$. Another vista may read as exact Lorentz symmetry forces experimental devise to observe a particle only along certain well-defined quantized and evolving trajectories in, say, $K$. Meaning, a measurement constitutes an act of physical interference at a given time. In other words, there is a physical coupling between a detection device and the normally wandering undetected classical particle at the time of measuring. Thus, one may sense a sort of distorted analogy between this deterministic and incomplete scenario and what the machinery of the QM formalism and its ambiguous quantum measurement procedure edify.

There is no denial that this prequantum scenario is incomplete and deficient in many respects, and as such cannot be taken too seriously. Besides, the nonlinear terms in the NLT of Section 3 often differ for the space or the time coordinate transformations; so, the above scenario does not typify our NLT. Yet, despite all that it succeeded in one thing: to show studying relativity in the context of NLT can lead to exciting and concealed potentialities never anticipated in the much more mundane SR. Undoubtedly there is still a long way ahead in our better understanding of the NLT and their overall impact on diverse areas of physics. Thus, the physics community is encouraged to get more involved in such (often neglected) explorations. For more details see Section 3.

Returning to SL, the overall idea of SL had been of minor academic interest, one reason being the lack of data, to a limited number of worldwide physicists for many decades. For example, an early, and rather off-putting, upper limit for the electron antineutrino SL anomaly $(\delta)$ was already available as early as the mid-1980s from the cosmological observations (dubbed SN1987a). The published result was $\delta=$ $\left(c_{\nu}-c_{\gamma}\right) / c_{\gamma}<4 \times 10^{-9}$ (see [1-5]). But situation changed unexpectedly when in September 2011 the OPERA Collaboration of CERN/Gran Sasso, following 3 years of data collection, announced the serendipitous detection of an unusually large muon neutrino $\left(\nu_{\mu}\right)$ superluminal anomaly [1-5]. All of a sudden it seemed a rather latent and speculative concept that turned into an experimental reality. The thrill or disbelieve typified the physics community mood for many tense months that followed thereafter (Sept 2011-July 2012). And as the result a large number of articles and publications appeared in the literature on SL, and expectedly a number of them provided a direct challenge to the OPERA experiment on technical grounds and demanded the CERN experimentalists to recheck their diverse measuring equipments thoroughly. In the late February 2012, CERN made a formal announcement on the misreading of the $\nu_{\mu}$ time-of-flight by the original OPERA experiment and traced the cause to a loose fiber optic, that in turn prompted a clock oscillator running too fast for the GPS synchronization.

The OPERA Collaboration conducted another round of, technically sound, muon neutrino experiment later on and claimed in a July 2012 article [1-5] that they no longer see marked signs of SL for such particles. The finalized verdict on SL, which in a sort nailed the muon neutrino SL in its coffin, or so it seemed, came about in a Dec 2012 archive article [15] by the OPERA collaboration supporting their earlier July 2012 conclusion. The latest OPERA data, which is an updated revision of their July data, is the most accurate we have to date. The latest data, which expectedly does not show a significant superluminal anomaly for the $\nu_{\mu}$, includes a more 
rigorous calculation of the experimental errors. It is worth noting that the first OPERA SL claim was so divisive that a number of other laboratories, also capable of measuring neutrino speeds, at Gran Sasso and elsewhere (like MINOS at the Fermilab which reported seeing a large SL anomaly in 2007 of the order $\delta=\left(c_{\nu}-c_{\gamma}\right) / c_{\gamma} \sim(5.1 \pm 2.9) \times 10^{-5}$ for the $\nu_{\mu}$ [1-5] but after reviewing their earlier data in 2012 precluded any SL [6]) got also involved in the game to either confirm or refute the OPERA findings. In fact, the Gran Sasso underground lab in Italy, located some $730 \mathrm{~km}$ away from CERN in Geneva, is also home to a number of other experimental facilities, besides OPERA, capable of making neutrino related measurements. These experiments go by the names: ICARUS, BOREXINO, and LVD. As it turned out, all the measurements of the $v_{\mu}$ velocity conducted by the aforementioned experiments in 2012, and utilizing the same pulsed-beam as OPERA, with pulse duration $\approx 3 \mathrm{~ns}$, do not confirm any marked SL. Meaning, in all experiments conducted so far the neutrino speed is consistent with the light speed to within the data uncertainties.

Ever since the mid-2012 and the latest CERN/Gran Sasso OPERA announcement of Dec 2012, the interest on SL is back to where it was before the controversial first OPERA statement. And, expectedly, the early astonishment, as well as the large number of articles written following the first OPERA announcement, has by now subsided considerably. In general, a fraction of the existing articles on SL are devoted to the alteration of the SR $E(P)$ dispersion relation, and only a smaller fraction of these deal directly with the deformation of the spacetime LT which is also the preferred methodology in this work.

Interestingly, even at this writing the idea of tiny $\mathrm{SL}$ is not entirely precluded from the field of particle physics. To see this better, it is worth discussing briefly what many of the known data tell us specifically on the observed magnitude of the neutrino superluminal anomaly. As we already did, one defines a dimensionless ratio $\delta=\left(c_{\nu}-c_{\gamma}\right) / c_{\gamma}$ as the standard for judging the magnitude of the superluminal anomaly; here $c_{\gamma}$ is the neutrino speed. Positive $\delta$ implies SL (not allowed by SR), negative $\delta$ implies subluminal and massive neutrinos (allowed), and $\delta=0$ refers to massless neutrinos (also allowed). In addition, it is evident that like any measured quantity $\delta$ is supplied also with both statistical and systematic errors, and the extent of these errors is vital for promoting SL which requires $\delta>0$, albeit small. The September 2011 OPERA result, which got everything started on SL [1-5], had to do with the claim of observing SL subsequent to detecting an overall statistically measured $\Delta t \approx 60$ ns earlier time of arrival for the $v_{\mu}$ beam compared to any light signal traveling the same distance $(\approx 730 \mathrm{~km})$. When used to assess the $\delta$ ratio, the result is $\delta \approx 2.5 \times 10^{-5}>0$. This $\delta$ value, which is by now repudiated, is associated with an unusually large SL anomaly. As shown soon, the four models in the first extended LT scenario discussed in Section 2 are by design flexible enough to reproduce any reasonably small value for the $\delta$ ratio (small enough to not mess up the subluminal predictions that are required to be very close to SR predictions) provided by a given experiment that is conducted, at least in principle, if not in practice, on any type of massive particle, and not just the neutrinos. If neutrinos are endowed with tiny SL anomaly, it must be also the case for any other elementary particle to allow for neutrinos to couple weakly with other SL particles while moving superluminally, thus the naïve logic for extending SL to all particles.

In Section 2, we will use the first scenario versions in few occasions to derive the large ratio for the $\delta$ (more precisely the ratio $c_{\nu} / c_{\gamma}$ ) that was first reported by OPERA to simply exemplify what our models are capable of doing. Obviously, by following the same steps one can make a prediction for the more recent $\delta$ values, like those provided by the July or the December 2012 OPERA Collaboration. As shown later on in Section 2, whatever $\delta$ value maybe it is simply a matter of adjusting certain free parameters in the models to predict the right $\delta$ value that comes closest to the experimental value, to within the data error range.

The July 2012 OPERA value for $\delta$, including also the data error range, is $\delta=[(2.7 \pm 3.1)$ (stat.) $+(3.4,-3.3)$ (syst.) $] \times$ $10^{-6}$, whereas the latest, and improved, December 2012 results for both $\delta$ and $\Delta t$ are $-1.8 \times 10^{-6}<\delta<2.3 \times 10^{-6}$ and $\Delta t=$ $0.6 \pm 0.4$ (stat.) \pm 3.0 (syst.) ns at $90 \%$ C.L. In 2011 the ICARUS experiment, using also $\nu_{\mu}$, provided an upper limit $\delta<1.25 \times$ $10^{-8}$, and in 2012 provided $\Delta t=0.3 \pm 4.9$ (stat.) \pm 9.0 (syst.) ns $[7,8]$, showing no marked signs of SL.

It is worth mentioning that there are planned experiments on neutrinos at the Fermilab for gaining more empirical information on the phenomenon of neutrino oscillations as commonly parameterized by a CP-violating phase, and a set of three angles. The NOVA project at the Fermilab, with a baseline of about 500 miles, will tackle these issues and more. Nova will begin collecting data this year. The NOVA experiments require an accurate knowledge of the neutrino speeds; so if there is any SL anomaly Nova should be able to discover it. Finally, as stressed earlier, there exists already a large literature on the subject of superluminality analyzed from different perspectives; for an incomplete sample of some of the more recent papers on SL and the LT modifications see [9-25].

Note. This author's stand on LT alteration subluminally dates back to his graduate study years where he conjectured that any type of massive and free particle incorporated in the standard model (the free leptons, quarks, gauge bosons, etc.) is endowed with its own intrinsic (and rest mass dependent) upper subluminal speed infinitesimally smaller than the light speed. At its maximal speed, a free SM particle reaches its finite upper energy and momentum in all proper inertial frames. There is room in this proposition to make it universal by invoking a single maximal energy scale (e.g., the Planck energy) to stand for the maximal attainable energy for all massive SM particles reaching their maximal speeds regardless of their identities. To the best of my knowledge, the novel idea of proper maximal speeds, as outlined above, was proposed first by this author in the late 1970s, while a graduate student, and thereafter underwent further maturation until about the mid-1980s. One of the consequences of the extended LT of that specific theory, which was primarily designed for subluminal speeds, was the extended framework allowed also for a dose of superluminality in a narrow range of speeds $>c$ for a peculiar class of particles not listed in the 
SM. The overall effort, however, remained latent after the late 1980 s heretofore with the recent claim of the $\nu_{\mu}$ SL detection by the first OPERA time-of-flight measurement. Two recorded preprints are available from those early years to corroborate this [26, 27]; a different exploration of this idea is also found towards the end of [28]. Unlike the 1970-80s, the concept of maximal speed has gained relative acceptances nowadays. In more recent times a number of authors have tackled the notion of subluminal maximal speeds by using diverse approaches. The most cited paper on maximal subluminal speed is an article by Coleman and Glashow [29]. By and large, although most published models on maximal speeds often use diverse approaches and technicalities, they have also a number of commonalities. As for this work, even though we are interchanging here the older notion of rest mass-dependent maximal subluminal speeds with the newer mass-dependent maximal superluminal speeds, the methods adopted in this note for modeling extended LT are not a continuance of the more involved proposals of the 1980s discussed in [26-28].

Lastly, soon after the claim of observing a large $v_{\mu}$ superluminal anomaly was made public by the OPERA Collaboration a number of theoretical physicists begin working on this Issue. Notable among them are Cohen and Glashow (CG) who conducted a thorough theoretical investigation on the $\nu_{\mu}$ energy loss during their $730 \mathrm{~km}$ long journey. And by using the earliest OPERA data in their formulation they concluded the alleged data had to be faulty. In the CG analysis [30] all the pertinent calculations were carried out for subluminal neutrinos; more on this later. Few months later, Bezrukov and Min Lee proposed a phenomenological Lorentz violating prescription for including superluminal neutrinos into their theory [31] and in the end reached the same rough conclusion as CG. For another novel theoretical approach to superluminal neutrinos see [32].

\section{Superluminal Extended Lorentz Transformations}

This section is an attempt to integrate the concept of superluminality (SL) into the relativistic theory through gentle deformations of the linear LT and in few cases invoke additional upper speeds beyond the light speed $c$. To keep this paper short, only two distinct scenarios coping with SL are studied, and obviously a number of other models are also possible. In the second scenario we reflect on preexisting constant superluminal speeds and include them in the extended LT construction, whereas in the first scenario upper superluminal speeds are derived explicitly via three realizations of this scenario (in fact there is also a fourth realization, our favorite one, discussed briefly at the end). In the second scenario we will introduce a superluminal speed $c^{*}$ in the LT that is neither the light speed $c$ nor the upper limit superluminal speed $\check{c}$ chosen as $\check{c}=c^{* 2} / c$, or more generally $\check{c}=$ $c^{\alpha} c^{*(1-\alpha)}$ where $\alpha$ is either an arbitrary constant or dependent on the boost velocity. In what follows though we set $\alpha=$ -1 . At the end of the second scenario we make specific predictions for the particle energies we should be observing at speeds $c$ and $c^{*}$ encompassing all massive particles including the neutrinos. These predictions are observed to be close to the energy range $\left(10^{13}-10^{20} \mathrm{eV}\right)$ observed in host of ultra-high energy cosmic ray detections. We also provide the modified $(E, \mathbf{P})$ relations for both scenarios, indicating possible deviations from SR. Needless to add that extending LT, albeit infinitesimally close to the standard LT, will forcibly have an impact on QM/QFT, the SM, string theory, local GR/QG and its diverse spacetime topologies, the standard practice of local spacetime slicing related by LT, Ads and de Sitter space, bubble nucleation speed limit $(c)$ and the theory of inflation, to name a few, are areas the LT distortion could brunt.

2.1. First Scenario and Its Four Realizations. In this first scenario we desire to retain the relativity principle and the light speed constancy intact, while also allowing superluminal boosts, but crafted in a manner that the altered Gamma factor $(\gamma)$ of the extended LT does not become a complex entity, at least in a limited range of SL, so as to avoid tachyon modes. In what follows we present four realizations of this scenario corresponding to 4 choices of a pivotal scale function $f(v)$ explained shortly, while keeping the light speed $c$ as the only constant in the LT. In the first realization after crossing the light barrier particles begin gradually behaving as having alike $M^{4}$ coordinates to two observers at rest in two inertial frames in uniform relative superluminal motion. Basically both observers begin reading almost equal time values and spatial coordinates for an event as if there is (almost) no boost. This is so because the subluminal LT linking two IF $K^{\prime}\left[\mathbf{r}^{\prime}, t^{\prime}\right]$ and $K[\mathbf{r}, t]$ will morph gradually to coordinate relations which imply $\left(\mathbf{r}^{\prime}, t^{\prime}\right) \rightarrow(\mathbf{r}, t)$, or $K^{\prime}\left[\mathbf{r}^{\prime}, t^{\prime}\right] \rightarrow$ $K[\mathbf{r}, t]$, for $v>1.9 c$. Technically, the difference between the two observer spacetime coordinate readings becomes smaller because the $\gamma$ factor approaches unity for boost speeds $v>$ $1.9 c$, or so. And there is no upper limit to SL, meaning any large SL speed would do after. This eccentric behavior stems directly from our particular choice of $f(v)$ for this realization.

In the second realization there will be a limited range of superluminality up to an upper SL speed; hence, superior boost velocities will no longer be admissible in the sense that the $\gamma$ factor becomes complex and rejected in this work. The third realization does a better job for the $\nu_{\mu}$, as used in last year's OPERA experiment, because the light speed crossing there will be a tiny gap of complex valued $\gamma$ in the region $0^{+}<\left(\nu_{\mu} / c\right)-1<2.4 \times 10^{-5}$, but afterward the superluminal particles become real particles with rapidly decreasing energies; here too there is an upper SL speed. The 4 th realization ranks the highest and is the closest of all to SR behavior in the subluminal region.

Let us begin with the familiar linear LT with the boost velocity $v$ taken in the $x$-direction:

$$
\begin{aligned}
x^{\prime} & =\cosh (\phi) x-\sinh (\phi) c t, \\
t^{\prime} & =\cosh (\phi) t-\frac{\sinh (\phi) x}{c} .
\end{aligned}
$$

It is trivial to show that the standard interval square is invariant under (1): $S^{\prime 2}=c^{2} t^{\prime 2}-r^{\prime 2}=S^{2}=c^{2} t^{2}-r^{2}$. 
The velocity composition rule involving $\phi(v)$ is $u^{\prime}=(u-$ $c \tanh (\phi)) /(1-\tanh (\phi) u / c)$. Next, we introduce a function $f(v) \geq 0$, assumed even in $v$ (because of reciprocity), and set $c \tanh (\phi)=v f(v)$ and impose the constraints: $f(0)=1$, $f(c)=1$. The new velocity composition rule (where $u=$ $d x / d t$ is the particle speed component in the $x$-direction in $K)$ which now reads

$$
u^{\prime}=\frac{u-v f(v)}{1-\left(u v f(v) / c^{2}\right)} .
$$

It is evident that for $u=c$ then $u^{\prime}=c$, likewise for $v=c$ then $u^{\prime}=-c$, exactly as is SR establishing the constancy of the light speed in $K$ and $K^{\prime}$; so $c$ is independent of the velocity of an observer, or the source emitting it. If a massive quantum particle is at rest in $K$, then a macroscopic observer in $K^{\prime}$ perceives its speed as $u^{\prime}=-v f(v)$, where $v$ is the boost relative speed for the classical IF. (Note that apart from thought experiments practical observations are forcibly made in one proper frame and not both simultaneously.) Utilizing these equations led also to the (E, P) dispersion relation(s). Manifestly, the $f(v)$ factor appears also in the usual Lorentz tensor $\Lambda_{\mu \nu}$ connecting $x_{\mu}^{\prime}$ and $x_{\mu}$ that we pass over. (An exciting case is when $u=v$ which in SR means $u^{\prime}=0$ but (2) implies $u^{\prime}=v(1-f(v)) /\left(1-v^{2} f(v) / c^{2}\right)$, and this result has a deep significance.)

The LT of (1) can now be cast in more common forms used hereafter:

$$
\begin{aligned}
& x^{\prime}=\gamma(v)[x-v f(v) t], \\
& t^{\prime}=\gamma(v)\left[t-\frac{v f(v) x}{c^{2}}\right] .
\end{aligned}
$$

An advantage of introducing the function $f(v)$ for the purpose of SL is seen after finding the new $\gamma$ factor:

$$
\gamma^{-2}(v)=1-\frac{v^{2} f^{2}(v)}{c^{2}} .
$$

If one allows the boost speed to go superluminal then in order to prevent complex values of $\gamma$ the function $f(v)$ (simply 1 in SR) must be smaller than 1 as $v$ exceeds $c$ such that $v f(v) \leq c$ (this is also a direct consequence of our definition $c \tanh (\phi)=v f(v)$ and the identity $\left.\cosh ^{2}(\phi)-\sinh ^{2}(\phi)=1\right)$. Invoking $f(v)$ has the advantage of providing an integrated formulation of the LT applicable at both sub- and superluminal boost speeds while avoiding tachyons; SL measurements are presumably performed on real particles only (regardless of how this is done in practice). In brief, while what is measured as the boost speed by macroobservers is $v$, what goes in the making of the LT and the velocity composition law for massive quantum particles is an effective speed $v_{\text {eff }}=v f(v) \leq c$ which differs infinitesimally from the rigid frame speed $v$ (see supplementary discussions at the end of this scenario). Broader forms of (3) and (4) can also be envisaged, for example, like $x^{\prime}=\gamma(v)[x A(v)-v B(v) t], t^{\prime}=$ $\gamma(v)\left[t A(v)-v B(v) x / c^{2}\right] \rightarrow \gamma^{-2}(v)=\left[A^{2}(v)-v^{2} B^{2}(v) / c^{2}\right]$. Here too the constancy of $c$ is respected, and $d S^{2}=d S^{2}$ provided $B(c) / A(c)=1$.

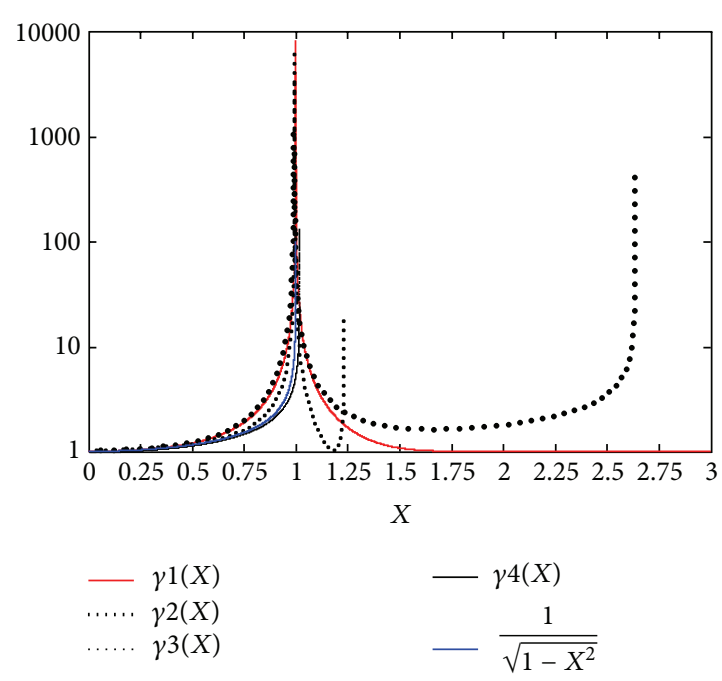

FIGURE 1: Four realizations of the $\gamma$ function versus $v / c$.

Although an exact way to model $f(v)$, so as to lead a consistent SR in the subluminal regimes, always exists in form of conditional constraints-that is, imposing $f(v)=1$ for all $v \leq c$ and a suitable $f(v)<1$ in the superluminal regimeswe will not pursue this exact route. Instead, we focus on finding suitable models for $f(v)$ thereafter viewed as a continuous function of $v$ covering all subluminal and a restricted or unrestricted range of superluminal speeds and then see what ensues. The motto is by pursuing this phenomenology that certain tiny differences with SR predictions may emerge in the subluminal regimes that could lead to observational consequences for certain particles, like perhaps the low-versushigh energy $\nu_{\mu}$ where some SL data are already available. The vital thing of course is to seek suitable forms of $f(v)$ that do not lead to unacceptable differences with SR in the subluminal regimes while at the same time capable of making specific predictions for any SL where traditional SR is moot.

In Figure 1 we study 4 distinct realizations of $f(v)$ that are neither unique nor exhaustive. The first realization does not provide any definite upper limit on SL, while the other three realizations predict definite upper superluminal speeds. We also stress that the first two realizations of $f(v)$, discussed first, are only for the purpose of illustration and showing variety, but they are also the least realistic ones in comparison to the 3rd and especially the 4th realization which are better suited for making predictions at all speed ranges, albeit not inclusive.

The first two realizations $f_{1}$ and $f_{2}$ are $f_{1}^{2}(v)=e^{X^{2}\left(1-X^{2}\right)}$, and $f_{2}^{2}(v)=e^{k X^{2}\left(1-X^{2}\right) /\left(1+k X^{6}\right)}$, where $k=0.34$ and $X=v / c$. The third realization is discussed in the next paragraph. The resulting first two gamma functions $\gamma_{1}$ and $\gamma_{2}$ are displayed in Figure 1; the blue solid line corresponds to the standard SR $\gamma=\left(1-X^{2}\right)^{-1 / 2}$. The solid red line is $\gamma_{1}$ of the first realization, and the black heavy dotted line is $\gamma_{2}$ of the second realization. As noted, there is no superluminal upper bound speed for $\gamma_{1}$ because beyond $v \sim 1.9 \mathrm{c}$, or so, the $\gamma_{1}$ factor is almost 1. This is not so in the second realization. In the latter case 
the $\gamma_{2}$ factor becomes complex beyond $v \sim 2.6406 c$, thus providing an upper superluminal speed of about 2.64c. Figure 1 also shows the amount of $\gamma_{1}$ and $\gamma_{2}$ deviations from the $\gamma$ factor of SR showing up around $v \sim 0.6 c$. If hypothetically such deviations ever existed for some types of particles, then it could provide a way of finding out in advance which particle can go superluminal by simply studying its relativistic behavior subluminally and how it deviates from SR. Of course more involved forms of $f(v)$, than the previous $f_{1,2}$ forms, could be sought so to bring the $\gamma_{1,2}$ much closer to the SR gamma values in all the subluminal region; so we leave this possibility open. It is worth stressing that while playing with numbers extreme care is needed for handling the $X$ values exceedingly close to 1 because all predictions are extremely sensitive to the tinniest change of $v$ in $f(v)$.

For the third realization the following polynomial form in $X$ is proposed $f_{3}(X)=1+k_{o} X^{2 n}\left(1-X^{2 n}\right)$ with the goal of generating a $\gamma_{3}$ curve closer to the SR blue line in Figure 1 than those of the previous two realizations while also accommodating SL. The final choice is $f_{2}(X)=1+0.25 X^{8}(1-$ $\left.X^{4}\right)$ giving $\gamma_{3}^{-2}=1-\left(X f_{3}(X)\right)^{2}$. The $\gamma_{3}$ variation is shown in Figure 1 as a thin black dotted line just above the SR blue line. As noted, here too one finds an upper superluminal speed that is much smaller than the one for the 2 nd realization. Interestingly, in this case the upper SL speed is $1.2357 c$. This case is interesting because it turns out that $\gamma_{3}$ is complex for all superluminal speeds smaller than $X_{\min }=1+2.4765 \times 10^{-4}$, but immediately after that $\gamma_{3}$ is basically large and real for all $X$ values found in the tiny interval $X_{\min }$ and $X_{\min }+6.633 \times$ $10^{-13}$ (this is the best we can do by using Mathcad) where the particle energy is very large and very rapidly decreasing with increasing $X$ ( $\mathrm{GeV}$ range neutrinos used in the first OPERA experiment may fit in this speed interval). For example, at $X=X_{\min }+6.633 \times 10^{-13}$ the huge $\gamma_{3}$ factor has gone down to a value $6.7109 \times 10^{7}$ which is also the very first number we are able to extract out of the Mathcad program. A $\nu_{\mu}$ with $40 \mathrm{eV}$ rest energy and $\gamma_{3} \sim 6.7109 \times 10^{7}$ yields an SL neutrino with energy $\sim 3 \mathrm{GeV}$, about the MINOS neutrino energy range. Of course a $40 \mathrm{eV}$ neutrino mass is ruled out observationally, and so a more powerful number crunching program is required to get the right $\gamma_{3}$ values for both the MINOS and the higher $\nu_{\mu}$ energy OPERA cases that could go along with the more realistic neutrino masses of less than $0.3-0.5 \mathrm{eV}$.

The $X_{\min }$ value, where superluminal real neutrinos begin showing up at ultra-high energies, implies $X_{\min }-1 \sim$ $2.476 \times 10^{-5}$ which is close to the result provided by the first controversial $v_{\mu}$ OPERA experiment $\delta=\left(c_{v_{\mu}}-c_{\gamma}\right) / c_{\gamma} \sim$ $2.5 \times 10^{-5}$ with an average energy $\sim 17 \mathrm{GeV}$. We note that the earlier value $k_{o}=0.249935$ in $f_{3}(v)$ was chosen so as to suite the first OPERA data. It is of course evident that $k_{o}$ must be adjusted appropriately to suite the latest $\delta$ OPERA data that we skip analyzing here.

Fourth Realization. We begin by discussing first a phenomenon that somewhat provided the inspiration to unveil in the next paragraph how we came across the 4 th realization. The event relates to how the clock reading in the 3rd realization gets altered from the standard SR reading. Consider two clocks located at $x_{1}$ and $x_{2}$ in the $K$ system (both reading $t$ ). An observer at rest in $K^{\prime}$ and using (4) uncovers

$$
\begin{aligned}
& t_{1}^{\prime}=\gamma_{3}(v)\left[t-\frac{v f(v) x_{1}}{c^{2}}\right], \\
& t_{2}^{\prime}=\gamma_{3}(v)\left[t-\frac{v f(v) x_{2}}{c^{2}}\right],
\end{aligned}
$$

or equally,

$$
\begin{aligned}
& t_{1}^{\prime}=t_{O / K}(v)-\gamma_{3}(v) \frac{v f(v) x_{1}}{c^{2}}, \\
& t_{2}^{\prime}=t_{O / K}(v)-\gamma_{3}(v) \frac{v f(v) x_{2}}{c^{2}}
\end{aligned}
$$

where $t_{\mathrm{O} / K}(v)=\gamma_{3}(v) t$ is the time showing on the clock at the origin of $K$ as sensed by the observer in $K^{\prime}$. Since $\gamma_{3} \neq \gamma_{\mathrm{SR}}$, one would expect a tiny time difference reading from what the same $K^{\prime}$ observer would have gotten in SR, but there is more. The second term $\gamma_{3}(v) v f(v) x_{1,2} / c^{2}$ represents an amount of clock "dephasing," to use a jargon, located at $x_{1}$ or $x_{2}$ in $K$, as sensed by the observer in $K^{\prime}$, compared to his time reading from the clock in $K$ located at the origin. Thus, one detects an infinitesimal mismatch between the aforementioned clock dephasing terms and the analogue SR term given by $\gamma_{\mathrm{SR}} v x_{1,2} / c^{2}$. Such tiny time mismatches provided the insight for us to propose something new in form of the 4 th realization where we seek only a very narrow window of SL close to $c$. Incidentally, if one uses the conditional constraint $f(v)=1$ for $v \leq c$, and $f(v)<1$ for $v>c$, then an independent possibility arises for finding $\gamma(v)$ in superluminal cases by simply surmising that for all $v>c$ the previous clock dephasing factors should satisfy the following relation $\gamma(v) v f(v) x_{1,2} / c^{2}=\gamma_{\mathrm{SR}}^{*} v x_{1,2} / c^{2}$ implying $\gamma(v) f(v)=\gamma_{\mathrm{SR}}^{*}$, where $\gamma_{\mathrm{SR}}^{*}=1 / \sqrt{v^{2} / c^{2}-1}$. In turn this relation leads to $\gamma(v)$ via $(5) ; \gamma(v)=\sqrt{\left(2 v^{2} / c^{2}-1\right)} /\left(v^{2} / c^{2}-1\right)$. The latter SL $\gamma(v)$, reaching a constant value $\sqrt{2}$ for $v \geq 3 c$, leads to new SL LT and a velocity composition rule. This form of $\gamma(v)$ though is not discussed any further.

Inspired by the previous briefing we have checked a variety of extended SL $\gamma(v)$ candidates to complete the 4 th realization. The right candidate could then be used as the archetype for limiting the SL to within only a narrow range of speeds extremely close to $c$. In what follows we exclusively focus on $f(v)$ forms given by $f_{4}(v)=1 / \sqrt{1+\lambda X^{n}}$, where $\lambda$ is small, $n \geq 2$, and $X=v / c$. Thus, the $\gamma$ factor of choice is $\gamma_{4}(v)=\left[1-X^{2} / \sqrt{1+\lambda X^{n}}\right]^{-1 / 2}$. This is shown in Figure 1 as a thin dark line for $\lambda=0.05$ and $n=2$. As seen, $\gamma_{4}$ is extremely close to $\gamma_{\mathrm{SR}}$ in the subluminal region for all $v \leq 0.97 \mathrm{c}$. Note also that $\gamma_{4}(v) \rightarrow \infty$ for definite superluminal values of $v$ very close to $c$ (e.g., it blows up at $v_{\max } \sim 1.0126 \times c$ for $\lambda=.05$ and $n=2$, or at $v_{\max }=1.0129 \times c$ for $\left.n=4\right)$, meaning that only a small superluminal region is permissible before $\gamma_{4}(v)$ turns complex and rejected. Moreover, notice that $f_{4}$ involves only one adjustable parameter $\lambda$ once $n$ is specified. Contrary to the earlier three realizations, with the $f_{4}(v)$ realization energy is not decreasing with increasing superluminal speed only 
allowed in the range $c \leq v \leq v_{\max }$; this is also a trend seen in the 2nd scenario.

Given the tininess of the $\delta$ anomaly, reported in host of neutrino experiments completed so far, we believe that this realization, especially if future experiments performed at high energies show a very small $\delta$, is more fitting for neutrino applications than the 3 previously discussed realizations (particularly the first two). While not fully pursued here, $\lambda$ can be adjusted accordingly to fit both the ICARUS and the OPERA superluminal anomalies. For example, for the ICARUS data a $\lambda$ value close to $2.5 \times 10^{-8}$ is the right value to predict their indicated $\delta$. Moreover, in this case the difference between $\gamma_{4}$ and $\gamma_{\mathrm{SR}}$ is so small that it is unlikely it could be detected in (almost) all subluminal regions.

To end, let us return to the 3rd realization and seek an expression for its energy-momentum dispersion relation. It is easy to show that if the momentum and the energy are defined, respectively, as $\mathbf{P}=m_{0} \mathbf{u} f_{3}(u) / \sqrt{1-u^{2} f_{3}^{2} / c^{2}}$, and $E=m_{o} c^{2} / \sqrt{1-u^{2} f_{3}^{2} / c^{2}}$, where $m_{o}$ is the rest mass, then $c^{2} P^{2} / E^{2}=u^{2} f_{3}^{2} / c^{2}$. By substituting this into $E$ the standard SR energy ensues $E^{2}=p^{2} c^{2}+E_{o}^{2}$. There is also another slightly different way of defining $E ; E=f_{3}(u) m_{o} c^{2} / \sqrt{1-u^{2} f_{3}^{2} / c^{2}}$. Then, through the previous $\mathbf{P}$ the liaison between altered $E$ and $E_{\mathrm{SR}}$ is $E^{2}=E_{\mathrm{SR}}^{2} f_{3}^{2}(u)$, where $E_{\mathrm{SR}}^{2}=p^{2} c^{2}+E_{o}^{2}$. Despite this simple relation linking $E$ and $E_{\mathrm{SR}}$, the resulting dispersion relation is complicated because of the way $f_{3}$ is related to both $E$ and $P ; f_{3}^{2}=1+k_{o}(P c / E)^{8}\left(1-P^{4} c^{4} / E^{4}\right)$, where $k_{o}=$ 0.249935 . We end by showing only the principal equation without trying to solve it to get $E(P) ; E^{14}=\left(p^{2} c^{2}+E_{o}^{2}\right)\left[E^{12}+\right.$ $\left.k_{o}(P c)^{8}\left(E^{4}-P^{4} c^{4}\right)\right]$.

Dialogue. Invoking the function $f(v)$ in the LT, besides affecting quantities like time dilation, length contraction, Doppler shifts, and so forth, raises also interpretational issues. So far the condition $f(v)<1$ was put to use in the context of phenomenology, leaving aside its unknown physical perceptive that may be involved (e.g., one could think of $f$ stemming from an exotic relevancy to the vacuum structure, preferred frame motion, universal rest frame and the ether, dark matter, quantum subtleties, and so forth). We have insisted throughout that $v$ is the common boost speed as measured and agreed on by two distinct (macroscopic, nonlocal) observers living in the global $K$ and $K^{\prime}$ IF in relative uniform motion. But what goes into the making of the physical modified LT linking both IF, and containing measurable quantum particles, is not the macroscopic boost $v$ but rather $v f(v)<c$, thus eradicating the option of redefining the boost speed as $v f(v)$ (i.e., recovering SR) and be done with it. If we were to use forthrightly the aforementioned conditional constraint on $f(v)$, then there is no bona fide issue of interpretation, at least in the subluminal regimes because $f(v<c)=1$. Throughout this scenario, though, we were resolute to extend $f(v)$ to both sub- and superluminal cases by having in mind the SM particles, like perhaps the neutrinos, as the physics protagonists for the spacetime events. There is no point of introducing $f(v)$ in the LT for classical objects (e.g., a rocket) since $f(v)=1$ for all $v<c$. Moreover, by relying on $f(v)$ it became possible to regard it as a handy numerical tool for assessing any would-be detectable signs of SL in advance for subluminal quantum particles at speeds nearing $c$. Thus far we justified the usage of $f(v)$ and presented 4 realizations of it, while the physics behind its existence is still missing. It is conceivable there is a more applied rationalization behind its existence via combining, for example, superluminality and resorting to the preferred frame concept so that $f(v)$ will also include the preferred frame speed $v_{p f}$, obviously giving rise to a peculiar $v_{p f}$ dependent velocity addition rule. Then the latter can be studied in the pivotal limit of $u=v$ for extracting valuable information (details omitted). To end, the physical origin of $f(v)$ remains fickle and left to future queries.

2.2. Second Scenario. In this scenario the deformation of the LT relies not on one but rather two superluminal speeds $c^{*}$ and $\check{c}=c^{* 2} / c$ which are assumed constants and independent of the boost velocity $v$. In addition, there is no longer an assertion for keeping the light speed $c$ universal in all IF. Instead, what is retained is the standard definition of an interval using $c$ and imposing its invariance under the modified LT. The metric is the usual Minkowski metric tensor $\eta_{\mu \nu}=\eta^{\mu \nu}=\operatorname{diag}(1,-1,-1,-1)$, itself a Lorentz invariant tensor (i.e., assuming the same form and values under the LT). The structure of relativistic mechanics in SR is designed to be Lorentz covariant under the global Lorentz coordinate transformations which are pertinent to our case as well. Finally, the emphasis here is generally on the Lorentz point of view somewhat differing conceptually from Einstein's SR. Consider the general form of standard LT having parallel axes between two inertial frames $K^{\prime}\left[\mathbf{r}^{\prime}, t^{\prime}\right]$ and $K[\mathbf{r}, t]$ in uniform relative motion with the velocity $\mathbf{v}$ pointing in an arbitrary direction, and as usual $\gamma=1 / \sqrt{1-v^{2} / c^{2}}$ :

$$
\begin{gathered}
\mathbf{r}^{\prime}=\mathbf{r}+\left[\frac{(\gamma-1)(\mathbf{r} \cdot \mathbf{v})}{v^{2}}-\gamma t\right] \mathbf{v}, \\
t^{\prime}=\gamma\left[t-\frac{\mathbf{r} \cdot \mathbf{v}}{c^{2}}\right] .
\end{gathered}
$$

The interval square $S^{2}=c^{2} t^{2}-r^{2}$ is obviously invariant under the previous linear coordinate transformations: $S^{\prime 2}=$ $S^{2}$. The spacetime linear transformations of (8) depict a boost in the $\mathbf{v}$ direction. The position vector $\mathbf{r}$ in the $K$ system and the constant boost velocity $\mathbf{v}$ can be chosen arbitrarily with a unit vector defined as $\mathbf{n}=\mathbf{v} / v$.

Note. The reduced LT, often found in textbooks, are derived from (8) by choosing $\mathbf{v}$ along the $z$ or $x$ direction. The inverse LT is found by replacing $\mathbf{v}$ by $-\mathbf{v}$ and interchanging the prime and the unprimed signs. When one speaks of the broader Lorentz transformations (BLTs), it means the combination of a boost along $\mathbf{v}$ and the rotations of the spatial coordinates. It so happens that physical laws of nature are covariant (i.e., unaffected in form) under the continuous spacetime BLT (i.e., boost plus rotation plus constant translation). Equally unaffected, as far as it is known, are the laws of nature under 
a combined discrete transformation labeled CPT (charge conjugation, parity transformation, and time reversal). Consequently, overall the laws of particle physics seem to be covariant under the combined BLT (more precisely the Poincaré transformations) and the CPT transformations.

We now take (8) and impart few alterations to them to wind up with (9) and (10). These changes incorporate superluminal speeds and allow for an infinitesimal violation of the light speed $c$, seen in two IF, while retaining the interval equality $S^{\prime 2}=c^{2} t^{\prime 2}-r^{\prime 2}=c^{2} t^{2}-r^{2}=S^{2}$. In order to speed up the staging we will stay away from many observer-related protracted debates, length contraction and time dilation discussions, and host of experimental debates that are typical of most homilies and articles on SR. Such topics are familiar in most SR discussions, forcibly applying to our scenario as well. The intention here is modest in that we merely desire to explore the current scenario through its modified LT. To end, causality is preserved throughout when using the modified LT in that a time-like separation between two events stays time-like after applying (9) and (10). The new proposed LT are

$$
\begin{gathered}
\mathbf{r}^{\prime}=\mathbf{r}+\left[\frac{(\alpha \gamma-1)(\mathbf{r} \cdot \mathbf{v})}{v^{2}}-\beta \gamma t\right] \mathbf{v} \\
t^{\prime}=\gamma\left[t-\frac{\mathbf{r} \cdot \mathbf{v}}{c^{* 2}}\right]
\end{gathered}
$$

Equations (9) and (10) are not unique. For example, one can propose two new and distinct equations as seen in (11a) and (11b) by simply changing (9) while leaving (10) intact, and so forth

$$
\begin{gathered}
\mathbf{r}^{\prime}=g(\mathbf{v}) \mathbf{r}+\left[\frac{(\gamma-1) \mathbf{r} \cdot \mathbf{v}}{v^{2}}-\beta(\mathbf{v}) \gamma t\right] \mathbf{v}, \\
\mathbf{r}^{\prime}=f(\mathbf{v}) \mathbf{r}+\left[\frac{f(\mathbf{v})(\gamma-1)(\mathbf{r} \cdot \mathbf{v})}{v^{2}}-\beta(\mathbf{v}) \gamma t\right] \mathbf{v} .
\end{gathered}
$$

One can show in the case (11b), for example, that by using velocity reciprocity we get $f=1$ and $\beta(\mathbf{v})=\beta(-\mathbf{v})$.

Hereafter we adhere to (9) and (10), where reciprocity implies $\alpha(\mathbf{v})=1, \beta(\mathbf{v})=\beta(-\mathbf{v})$. The parameter $\beta$ in (9) is assumed positive and constant, and $c^{*}$ is a SL speed scale $>c$. No restriction is imposed on $c^{*}$ to be a constant; it can be, for example, an even function of the boost velocity $\mathbf{v}$. Yet, for simplicity sake we will take it to be a constant. Like (8), (9) and (10) are also linear relations between $x_{\mu}^{\prime}$ and $x_{\mu}$, implying that if $\left(d S^{\prime}\right)^{2}=(d S)^{2}$ then $S^{\prime 2}=S^{2}$, and vice versa. As for neutrinos, we surmise that they satisfy $(d S)^{2}>0$, dubbed time-like in SR. The $K / K^{\prime}$ local clocks located at $\mathbf{r}^{\prime}$ and $\mathbf{r}$ are synchronized at origin $t=t^{\prime}=0$ and $\mathbf{r}^{\prime}=\mathbf{r}=0$, or when passing through each other.

Subsequently, by demanding the equality of $S$ and $S^{\prime}$ intervals under the transformations of (9) and (10), we find $\beta$ and the new $\gamma$ factor. The results are $\beta=\left(c / c^{*}\right)^{2}$ and $\gamma^{-2}=1-c^{2} v^{2} / c^{* 4}$. It is marked that unlike in SR the new gamma factor is finite at $v=c$, albeit large. Yet $\gamma$ becomes singular at the SL speed $\check{c}=c^{* 2} / c$ which is to be recognized as the maximum allowed SL for which $\gamma \rightarrow \infty$ is still real.
Prior to addressing the free particle energy and momentum (dispersion) relation and its derivation, we first derive the modified expression for the velocity composition rule. Straightforward calculation, using (9), (10), and the usual definitions $\mathbf{u}=d \mathbf{r} / d t$ and $\mathbf{u}^{\prime}=d \mathbf{r}^{\prime} / d t^{\prime}$ gives

$$
\mathbf{u}^{\prime}=\frac{\gamma^{-1}\left\{\mathbf{u}+\left[(\gamma-1)\left(\mathbf{u} \cdot \mathbf{v} / v^{2}\right)-\gamma\left(c / c^{*}\right)^{2}\right] \mathbf{v}\right\}}{1-\left(\mathbf{u} \cdot \mathbf{v} / c^{* 2}\right)}
$$

with $\gamma^{-1}=\sqrt{1-c^{2} v^{2} / c^{* 4}}$. Thus, the parallel and perpendicular components of $\mathbf{u}$ about the direction of $\mathbf{v}$ are

$$
\begin{gathered}
u_{\|}^{\prime}=\frac{u_{\|}-v\left(c / c^{*}\right)^{2}}{1-\left(u_{\|} v / c^{* 2}\right)}, \\
u_{\perp}^{\prime}=\frac{u_{\perp}}{\gamma\left(1-\left(u_{\|} v / c^{* 2}\right)\right)} .
\end{gathered}
$$

The velocity composition rules in (13) are loaded in content and entail important, and sometimes odd, numerical asymmetry at upper speeds that we must skip. Instead, we provide a self-explanatory sample of few key upper speed components along $\mathbf{v}$. This limited sample also reveals the peculiar possibility of having an infinite speed for $u_{\|}^{\prime}$ at certain values of $u_{\|}$or $v$, which are justly absent in SR.

(1) $u_{\|}= \pm c \rightleftarrows u_{\|}^{\prime}= \pm c$, for all $v$. This result is identical to SR.

(2) $v= \pm c \rightarrow u_{\|}^{\prime}=\mp c+\Delta_{ \pm}$, where $\Delta_{ \pm}=\left(c^{* 2}-c^{2}\right)\left(u_{\|} \pm\right.$ $c) /\left(c^{* 2} \mp u_{\|} c\right)=c(\check{c}-c)\left(\left(u_{\|} / c \pm 1\right) /\left(\check{c} \mp u_{\|}\right)\right)$. The often nonzero $u_{\|}$dependent $\Delta_{ \pm}$provides a measure of $u_{\|}^{\prime}$ deviation in the $K^{\prime}$ IF from the expected $\mp c$ in SR.

(3) $v= \pm \check{c} \rightarrow u_{\|}^{\prime}=\mp c$, for all $u_{\|}$, where $\check{c}=\left(c^{* 2} / c\right)$ is the maximum allowed superluminal speed appearing in the $\gamma$ factor.

(4) $u_{\|}= \pm \check{c} \rightarrow u_{\|}^{\prime}= \pm \check{c}+\left(v\left(\check{c}^{2}-c^{2}\right)\right) /(\check{c}(c \mp v))$.

(5) $u_{\|}= \pm c^{*} \rightarrow u_{\|}^{\prime}= \pm c^{*}+\left(v\left(c^{* 2}-c^{2}\right)\right) /\left(c^{*}\left(c^{*} \mp v\right)\right)$.

(6) $v= \pm c^{*} \rightarrow u_{\|}^{\prime}=\mp c^{*} \pm\left(c^{* 2}-c^{2}\right) /\left(c^{*} \mp u_{\|}\right)$.

So far all known particles are observed while moving subluminally. So to derive the $E(\mathbf{P})$ dispersion we will retain the SR rest energy $E_{\text {rest }}=m_{o} c^{2}$ and propose the subsequent forms for the energy and the momentum:

$$
\begin{aligned}
& E=\frac{E_{\text {rest }}}{\sqrt{1-\left(c^{2} v^{2} / c^{* 4}\right)}}, \\
& \mathbf{P}=\frac{m_{0} \mathbf{v} \zeta(v)}{\sqrt{1-\left(c^{2} v^{2} / c^{* 4}\right)}} .
\end{aligned}
$$

Here $\zeta(v)$ is an adjustable function (see the end of this section) to appear in the $E(\mathbf{P})$ relation. Focusing on (14) it is now trivial to find the relation between the velocity and the energy (recall in the quantum picture the velocity is the group velocity of the wave packets):

$$
v^{2}=\left(\frac{c^{* 2}}{c}\right)^{2}\left(1-\frac{E_{\text {rest }}^{2}}{E^{2}}\right)=\check{c}^{2}\left(1-\frac{E_{\text {rest }}^{2}}{E^{2}}\right) .
$$


Thereafter we denote by $E_{m}=M c^{2}$ the energy of all massive particles reaching speed $v=c^{*}$. For the neutrinos we symbolize this energy by $E_{m v}=M_{\nu} c^{2}$ where normally $M_{\nu} \ll M$. Next, we take $M$ to be a universal mass scale for all massive particles (plausible candidates are the Planck mass $M_{P}$ or a GUT type mass scale), while exempting neutrinos for now because $M_{\nu}$ may depend on the $v$ flavor. The results for upper speeds and the $\gamma(v)$ factor are

$$
\begin{gathered}
c^{*}=\frac{c}{\sqrt{1-\left(m_{0}^{2} / M^{2}\right)}} \longrightarrow \frac{c^{*}-c}{c}=-1+\frac{1}{\sqrt{1-\left(m_{0}^{2} / M^{2}\right)}}, \\
\check{c}=\frac{c}{1-\left(m_{0}^{2} / M^{2}\right)}, \\
\gamma^{-1}=\sqrt{1-\left(1-\frac{m_{0}^{2}}{M^{2}}\right)^{2}\left(\frac{v^{2}}{c^{2}}\right)}
\end{gathered}
$$

It is now simple to show $\gamma_{c^{*}}=M / m_{0}$ and $\gamma_{c}=$ $M /\left(m_{o} \sqrt{2-m_{o}^{2} / M^{2}}\right)$. When $v=c, E_{c}=M c^{2} / \sqrt{2-m_{o}^{2} / M^{2}}$ and is finite. For most particles $M \gg m_{o}$ and the latter equation is approximately $E_{c} \approx M c^{2} / \sqrt{2}$. Equations (17a), (17b), and (17c) also specify for a particle of mass $m_{o}=M$ that intriguing phenomena can occur; since the math is trivial we leave it to the reader to survey this case.

Let us now use $E_{c}$ so as to predict the energy of few massive particles at $v=c$, which is the ultimate subluminal relativistic frontier one can explore. To do that, we note that the earlier assertion for setting $M=M_{P}$ at $v=c^{*}$ was valid for only free particles moving in the vacuum. This universal setting has to be altered for the ultra-relativistic charged cosmic rays (URCR) traveling long distances and interacting along the way with the $2.7 \mathrm{~K}$ photons in the cosmic microwave background (CMB). Thus, the earlier universal scale $M$ is to be changed now to a rest-mass dependent scale $M\left(m_{o}\right)$ to take into account particle couplings with the CMB photons. To make contact with a host of URCR observations, we set $M\left(m_{o}\right)=\sqrt{m_{0} M_{P}}$. The latter phenomenological form, which leads to $E_{c} \approx \sqrt{E_{m} E_{\text {rest }} / 2}$, makes particle energy predictions at $v=c$ reasonably close to a host of observed URCR energies; here $E_{m}=c^{2} \sqrt{m_{o} M_{P}}$.

Now, for a proton one finds $E_{c} \approx 2.4 \times 10^{18} \mathrm{eV}$ (as a reminder the GKZ cut-off is $\approx 4 \times 10^{19} \mathrm{eV}$ ), for an Iron nuclei $E_{c} \approx 1.22 \times 10^{19} \mathrm{eV}$, for the muon $E_{c} \approx 8 \times 10^{17} \mathrm{eV}$, and for an electron $E_{c} \approx 5.5 \times 10^{16} \mathrm{eV}$. If cosmic rays (CRs) of greater energies than the GKZ cut-off are observed [33,34], and if one is able to identify the particle species in such CR, then a mass scale $M>\sqrt{m_{o} M_{P}}$ may be considered, obviously dwindling any would-be superluminal anomaly. Thus far the highest, and extremely scarce, cosmic ray energy ever reported is $3 \times 10^{20} \mathrm{eV}$.

Let us consider the neutrinos. Needless to say there is neither a satisfactory understanding of the neutrino masses, nor direct experimental data on individual neutrino masses (indirect estimates do exist from the phenomenon of neutrino oscillation that we disregard). Instead, what we have, through cosmic observations, is the total neutrino mass upper limit $\sum m_{v} \approx 0.28-0.5 \mathrm{eV}$ [35]. If we insist on extending $M=\sqrt{m_{o} M_{P}}$ to neutrinos then for a neutrino of rest energy $1 \mathrm{eV}$ we find $E_{c}(\nu) \approx 8 \times 10^{13} \mathrm{eV}$. Following six years of observation, the Pierre Auger Observatory reported recently not observing neutrino cosmic events in the range $10^{17}$ to $10^{20} \mathrm{eV}$ [36]; so for now our prediction holds. Also another plausible mass scale to try is $M=M_{\mathrm{GKS}}=4 \times 10^{19} \mathrm{eV}$ which is the GKS cut-off energy.

To complete the task of estimating $\delta=\left(c_{\gamma_{\mu}}(E)-c_{\gamma}\right) / c_{\gamma}$, we choose a purely phenomenological expression for $M_{v_{\mu}}$ set as: $\left.M_{v_{\mu}}=(\alpha / 2 \pi) m_{e}(\sim 581) \mathrm{eV} / \mathrm{c}^{2}\right)$, where $m_{e}$ is the electron rest mass. This setting gives the value $\delta \sim 1.25 \times 10^{-8}$ for the multi-GeV neutrinos indicated recently by ICARUS $[7,8]$. The ICARUS result is more consistent with other past $\delta$ findings, all repudiating the early SL OPERA claim. Next consider (17a), it indicates that for neutrino species of low rest mass and GeV range energy, the energy dependency in $c_{\nu_{\mu}}(E)$ can be safely ignored. Thus, we approximate $c_{\nu_{\mu}}(E) \approx c^{*}$ and assume $m_{o v_{\mu}}^{2} / M_{v_{\mu}}^{2} \ll 1$, consequently $\delta \approx(1 / 2) m_{o v_{\mu}}^{2} / M_{v_{\mu}}^{2}$ or $m_{o v_{\mu}} / M_{v_{\mu}} \approx \sqrt{2} \delta$.

Now, by using the ICARUS upper $\delta$ value we find the estimated $\nu_{\mu}$ rest energy: $E_{o_{\nu_{\mu}}} \leq 0.092 \mathrm{eV}$; as a result for three assumed equal mass neutrino flavors we find $\sum m_{v} \approx 0.28 \mathrm{eV}$ which is consistent with the cosmological findings [35].

Note. A back-of-the-envelope estimate of the neutrino masses may be performed by devising an energy-time look-alike uncertainty principle: $\left|m_{v_{\mu}}\left(v_{v_{\mu}}-c_{\gamma}\right) c_{\gamma} \Delta t_{v_{\mu}}\right| \approx \hbar / 2$ where $\Delta t_{v_{\mu}}$ is the $\nu_{\mu}$ 's arrival time difference with respect to the light arrival time. By using the first contentious OPERA values $\Delta t_{v_{\mu}} \sim 60 \mathrm{~ns}$, and $\left(v_{v_{\mu}}-c_{\gamma}\right) / c_{\gamma} \sim 2.4 \times 10^{-5}$ the resulting muon neutrino mass is $m_{v_{\mu}} \sim 2.26 \times 10^{-4} \mathrm{eV} / \mathrm{c}^{2}$, but by, e.g., using instead the last July OPERA data $\Delta t_{\nu_{\mu}} \sim 6.5 \mathrm{~ns}$ and $\left(v_{v_{\mu}}-c_{\gamma}\right) / c_{\gamma} \sim 2.7 \times 10^{-6}$ (omitting all the data uncertainties) then $m_{v_{\mu}} \sim 0.0185 \mathrm{eV} / \mathrm{c}^{2}$. The same format for the SN1987a [1-5] with $\left(c_{v_{e}}-c\right) / c \sim 10^{-9}$ and $v_{e}$ arrival time of 12 seconds ahead of the light arrival time gives $m_{v_{e}} \sim 2.7 \times 10^{-8} \mathrm{eV} / \mathrm{c}^{2}$ for the electron neutrino mass. Although very small masses they are not necessarily in conflict with most observational upper limits, but may spell trouble for the $v_{1}, v_{2}, v_{3}$ oscillation data providing various $\Delta m_{i j}^{2}$ values typically $\sim 10^{-3}-10^{-4} \mathrm{eV}^{2} / \mathrm{c}^{4}$. A way to surmount this is to lower the SN1987a ratio $\left(c_{v_{e}}-c\right) / c \approx$ $4 \times 10^{-9}$ by orders of magnitude. For example, a ratio $\sim 10^{-15}$ gives a much larger $m_{v_{e}} \sim 0.0274 \mathrm{eV} / \mathrm{c}^{2}$, this mass may not be prohibitive due to subtleties existing in the multitude of time-of-flight estimations in the SN1987a analyses. A sturdy constraint on $\mathrm{TeV}$ range neutrinos has already been provided by the IceCube Collaboration $\left(c_{v}-c\right) / c \sim 1.7 \times 10^{-11}$.

Yet, despite obtaining reasonable neutrino rest mass values, by using the ICARUS upper limit on $\delta=\left(c_{v}-c\right) / c$, one may argue why not invoke a $\mathrm{GeV}$ range mass scale, instead 
of the previous $M_{v_{\mu}}=581 \mathrm{eV} / \mathrm{c}^{2}$ that is far below the $\mathrm{GeV}$ range of most observed neutrinos. Let us look briefly at this possibility by setting $E_{m v_{\mu}} \approx 10 \mathrm{GeV}$, while keeping $E_{o v_{\mu}} \approx$ $0.1 \mathrm{eV}$, and compute $\delta$. The result is $\delta \approx 5 \times 10^{-23}$. Visibly this value is orders of magnitude smaller than the ICARUS and the SN1987a upper limits on $\delta$. Likewise, for the SN1987a with $10 \mathrm{MeV} \nu_{\mu}$, we find $\delta \approx 5 \times 10^{-17}$, again way below the reported upper limit.

The new issue now is whether a very tiny $\delta$ as aforementioned contradicts experiments, for example, the ICARUS experiment $[7,8]$. The ICARUS Collaboration concludes that their experiment does not indicate any statistically significant time-of-flight deviation from the unperturbed spectrum $\delta=0$. From our side the message is this: we have a format that can be tailored, by simply adjusting $M$ at $v=c^{*}$ accordingly, to satisfy any small or larger observed $\delta$, including the by now discarded OPERA $\delta$ value not discussed here because it requires a much larger $m_{o} / M$ ratio in comparison to ICARUS, so making it hard to justify physically. At this writing though one cannot tell whether $\delta \approx 10^{-9}$ is more desirable or a tiny $\delta \approx 10^{-20}$. These are issues that must be decided on when more precise SL data become available. What we can say for now is in case very tiny $\delta$ values are the outcome of future neutrino experiments then there is no simple bulk reasoning why neutrino species should be distinguished from the rest of the massive particles. That is, in principle one is free to set $M_{\gamma_{\mu}}=M_{P}$, or possibly choose any other larger or smaller (GUT-like) mass scale, and be done with it.

A very tiny $\delta$ is not without benefits, for one no conflict will arise from the phenomenon of pair bremsstrahlung predicted by the mainstream physics, with the cross section scales as $\delta^{3}$. Consequently, the earlier objection of Cohn and Glashow, which was based on a detailed $v$ pair bremsstrahlung analysis in [30-32], to the large value of $\delta$ when first reported by OPERA, is no longer of an issue for the much smaller $\delta$ indicated by the latest OPERA data reported last July and December. While detecting very small (positive) $\delta$, where a tiny SL is also allowed, is beyond the current capabilities, it may have a relevancy in large scale probing of the universe. Convoluted forms of nonlinear LT admitting SL, in juxtaposition with QM, may provide an entirely different understanding of $\delta$ and its smallness.

We conclude this section by providing the $E(\mathbf{P})$ dispersion relation for the second scenario only. Returning to (14) and (15) it is trivial to show $\left(E / c^{2}\right) \mathbf{v} \zeta(v)=\mathbf{P}$ that is then used for finding the $E(\mathbf{P})$ dispersion relation:

$$
E^{2}=\left(\frac{c^{2}}{\zeta(v) \check{c}}\right)^{2} P^{2}+m_{o}^{2} c^{4} .
$$

The remaining task is modeling $\zeta(v)$ and then substituting $v$ from $(16)$ in $\zeta(v)$. In general this could lead to a complicated $E(\mathbf{P})$ dispersion relation. To avoid unnecessary complexities we consider only two simple forms for $\zeta(v):(\mathrm{a}) \zeta(v)=c / \check{c}$ and (b) $\zeta(v)=1$. Using (a) provides the SR dispersion relation of (19a), while using (b) gives (19b),

$$
\begin{gathered}
E^{2}=c^{2} P^{2}+m_{o}^{2} c^{4}, \\
E^{2}=\left(\frac{c^{2}}{\check{c}}\right)^{2} P^{2}+m_{o}^{2} c^{4} .
\end{gathered}
$$

It is now straightforward to express $E$ and $\mathbf{P}$ via (15) and (17a), (17b), and (17c) in terms of $m_{o}$ and $M$ for either $\zeta(v)$ option. The case $M=M_{P}$ is special because one can write

$$
\frac{-m_{o}^{2}}{M^{2}}=\frac{-G m_{o}^{2}}{r_{c} m_{o} c^{2}}=\frac{U_{\mathrm{grav}}\left(r_{c}\right)}{m_{o} c^{2}}
$$

where $U \equiv-G m_{o}^{2} / r_{c}$ is the gravitational self-energy of a particle at its Compton radius $r_{c}=\hbar / m_{o} c$.

Finally, a sensible question comes to mind: what if there is no SL at all, which is also consistent with all existing neutrino data? In this case a reformulation of the 2nd scenario can be sought by purging $\check{c}$ and, as an option, treating $c^{*}$ as the maximum subluminal speed(s) for massive particles.

\section{Nonlinear Lorentz Transformations (NLT): A Personal Outlook}

3.1. Prelude. Prior to discussing scores of technical details related to various upcoming NLT models proposed in this section let us, to set the stage, construct the first highly nonlinear LT for linking spacetime coordinates of two inertial frames in uniform relative motion (thereafter taken along the $x$ axis). The novel construct will have to include few requirements that could serve as an inspiration for extending the linear LT to other exotic type NLT. These requirements are as follows.

(1) The local NLT should have a placid dependency on an "environmental" length scale $l$ encompassing the relativistic system under study (be it characterizing a string length, the quark confining region inside the hadrons, a linear scale of a cavity in the lab, or perhaps related to the Hubble constant and the universe). This is a Machian type inspiration, per se, meant for spacetime and not the mass.

(2) When the scale $l$ is large compared to the probing region under study (e.g., $l \gg c t$ ) the standard LT with tiny nonlinear corrections must ensue.

(3) Unlike the linear LT cases the NL transformations (rotations excluded) are to be atypical in that they should not admit standard Lorentz tensorial type connections $x_{\mu}^{\prime}=\Lambda_{\mu}^{v} x_{\nu}$ and $p_{\mu}^{\prime}=\Lambda_{\mu}^{\nu} p_{\nu}$ from the onset; yet these become viable connections whenever nonlinearities turn into small perturbation.

(4) For vanishing boost speed $x^{\prime \mu}=x^{\mu}$ must result.

(5) The inverse NLT $(x, t) \rightarrow\left(x^{\prime}, t^{\prime}\right)$ is to be distinct from the NLT $\left(x^{\prime}, t^{\prime}\right) \rightarrow(x, t)$, the distinction could 
be as extreme as the difference between, say, a log function and an exponential. Yet, for probing regions much smaller than $l$ all nonlinear peculiarities must dwindle explicitly and the standard SR should be recovered (modulo small nonlinear correction terms $\approx l^{-1}$ ).

(6) While an observer in a stationary inertial system $K$ can view a plane wave as $\sin (\omega t+k x)$, an observer in the uniformly moving IF $K^{\prime}$ could perceive the same wave differently (this is a general statement though, since for large $l$ the difference with SR is only marginal), and vice versa. Thus, one expects to find intricate spacetime interrelations between the angular frequencies (beyond the SR result $\omega^{\prime}=$ $\omega \sqrt{(1+v / c) /(1-v / c)})$ and the wave numbers in $K$ and $K^{\prime}$ systems. These, in turn, could have novel cosmological implications, involving now small nonlinear terms at the level of $1 / l$ in the wave phase proportional to $t^{\prime 2}, x^{\prime 2}, x^{\prime} t^{\prime}$, and the higher powers.

(7) The sought NLT will have to predict either exactly or almost exactly the constancy of the light speed $c$. In other words, the terms proportional to $1 / l$ should be all tiny compared to the linear terms. We desire, and indeed expect, besides a tiny Lorentz symmetry violation, also observing small anisotropy in the speed addition rule resulting from the NLT. Finally, it is desirable to focus on designing those NLT leading to velocity addition rules that are simple enough to unable one to study the settings for which SL can become a possibility when the boost speed (or the particle speed) reaches $c$.

The search for an NLT, satisfying the previous tips, led to the following NLT in the Cartesian coordinates:

$$
\begin{gathered}
x^{\prime}=\frac{\gamma l c}{v} \ln \left[1+\frac{v}{c} \frac{(x-v t)}{l}\right], \\
t^{\prime}=\frac{\gamma l}{v} \ln \left[1+v \frac{\left(t-\left(v x / c^{2}\right)\right)}{l}\right] .
\end{gathered}
$$

The previous NLT do not apply to all spacetime events if $l$ is not large enough, and they apply only to cases where both sums of the terms in the log functions of (21a) and (21b) are positive (i.e., excluding complex primed coordinates). Obviously, if $l$ is large there is no spacetime restriction. The inverse transformations are of course exponentials:

$$
\begin{array}{r}
x=\gamma^{2} l\left[c \frac{\left(e^{\left(v x^{\prime} / \gamma c l\right)}-1\right)}{v}+e^{\left(v t^{\prime} / \gamma l\right)}-1\right], \\
t=\gamma^{2} l\left[\frac{e^{\left(v t^{\prime} / \gamma l\right)}-1}{v}+\frac{e^{\left(v x^{\prime} / \gamma c l\right)-1}}{c}\right] .
\end{array}
$$

To order $1 / l$ the NLT in (21a) and (21b) turn to the standard LT plus small quadratic corrections:

$$
\begin{aligned}
x^{\prime} & \approx \gamma\left[x-v t-\frac{v}{2} \frac{(x-v t)^{2}}{c l}+\mathcal{O}\left(\frac{1}{l^{2}}\right)\right] \\
& \approx \gamma(x-v t)\left[1-\frac{v}{2} \frac{(x-v t)}{c l}+\cdots\right], \\
t^{\prime} & \approx \gamma\left[t-\frac{v x}{c^{2}}-\frac{v}{2} \frac{\left(t-\left(v x / c^{2}\right)\right)^{2}}{l}+\mathcal{O}\left(\frac{1}{l^{2}}\right)\right] \\
& \approx \gamma\left(t-\frac{v x}{c^{2}}\right)\left[1-\frac{v}{2} \frac{\left(t-\left(v x / c^{2}\right)\right)}{l}+\cdots\right] .
\end{aligned}
$$

The exact velocity addition law, by utilizing directly (21a) and (21b), reads

$$
u^{\prime}=\left[\frac{u-v}{1-\left(u v / c^{2}\right)}\right]\left[\frac{l+v\left(t-\left(v x / c^{2}\right)\right)}{l+(v(x-v t) / c)}\right] .
$$

As noted, the first bracket in (24) is identical to the SR result, while the second (spacetime dependent) bracket is the total correction there is in this model to the SR velocity addition law.

The next task for us is to study in brief (24) in the ultrarelativistic regimes. A short summary is as follows. When $v=$ $c \rightarrow u^{\prime}=-c(l+(c t-x)) /(l-(c t-x))$, but since causality requires $c t>x$ then the latter result may be a hint of a particle spacetime dependent superluminality. But for the case $v=$ $-c \rightarrow u^{\prime}=c$, as in SR. On the other hand, for $u=c$ (and setting $x=c t) \rightarrow u^{\prime}=c$, as in SR. But for $u=-c$ (and setting $x=-c t) \rightarrow u^{\prime}=-c(l+v t(1+v / c)) /(l-v t(1+v / c))$. Hence, altogether we observe an anisotropy for only 2 out of 4 ultrarelativistic cases, that is, for $v=c$ and $u=-c$. For a particle at rest in $K$ (i.e., $u=0)$ then $u^{\prime}=-v\left[\left(l+v\left(t-v x / c^{2}\right)\right) /(l+\right.$ $v(x-v t) / c)]$, and the latter bracket is the correction to SR. Although omitted here, interested readers may want to try the standard quadratic interval, the line element and the proper time links amid the $K$ and $K^{\prime} \mathrm{IF}$, and lastly try to obtain the rather involved $E(\mathbf{P})$ dispersion relation.

Later we tackle a bit the notion of deformed metric, owing to the NLT of (21a) and (21b), as perceived in one of the two inertial frames in relative uniform motion. Let us pretend the observer in the moving IF $K^{\prime}$ perceives its own metric as Minkowskian: $\eta_{\mu \nu}=\operatorname{diag}(1,-1,-1,-1)$, where the usual quadratic line-element: $d S^{\prime 2}=c^{2} d t^{\prime 2}-d x^{\prime 2}-d y^{2}-d z^{2}=$ $g_{\mu \nu} d x^{\mu} d x^{\nu}$ applies. If so, then when transformed to the $K$ IF coordinates $(x, y, z, t)$ this metric is a function: $g_{\mu \nu}(x, y, z, t)$. The full metric $g_{\mu \nu}(x, y, z, t)$ as perceived in $K^{\prime}$ is too involved to be shown here, instead we provide the $g_{\mu \nu}$ metric involving only corrections up to first order in $1 / l$ (though not done here there is a benefit to invoke the vierbein approach $e^{a}$ for conducting the full metric study: $\left.g_{\mu \nu}=\eta_{a b} e_{\mu}^{a} e_{\nu}^{b}\right)$. The nonvanishing elements of this metric are $g_{00}=1-2 v[t(1-$ $\left.\left.v / c+v^{2} / c^{2}\right)-v x / c^{2}\right] /(l(1-v / c)), g_{11}=-1-2 v[v t-x(1-v / c+$ $\left.\left.v 2 / c^{2}\right)\right] /(l c(1-v / c)), g_{22}=g_{33}=-1$, and there are also two 
tiny off-diagonal elements $g_{01}=g_{10}=2 v^{2}(c t-x) /\left(l c^{2}(1-\right.$ $v / c)$ ) vanishing when $x$ is on the future light-cone.

Note. What is found here is somewhat proverbial to the procedure followed in the linearized GR yielding a Minkowski metric $\eta_{\mu \nu}$ plus a small tensor $h_{\mu \nu}$ correction that is often employed to describe the phenomenon of gravity waves. Yet, and only for fun, the affine connection, the Ricci tensor, and evidently the scalar curvature are all zero if the previous approximate metric, which is linear in $x$ and $t$, is used.

At this stage two interesting cases arise for the previous approximate metric: (1) when a (massless) particle worldline is $x=c t$, and (2) when the worldline of a massive particle is $x=v t$ (i.e., the particle is at rest in the uniformly moving IF $K^{\prime}$ ). For case (1) the resulting metric elements are $g_{00}=$ $-g_{11}=1-2 v t(1-v / c) / l=1-2 v x(1-v / c) / c l, g_{01}=g_{10}=0$ and $g_{22}=g_{33}=-1$. (Once again it is amusing, but not justifiable, to compare the derived $g_{00}$ in case (1) with the classic prediction of GR in the weak (gravity) field limit $g_{00}=1+$ $2 \Phi / c^{2}$, whereby $\Phi=-v x(c-v) / l$ is the gravitational potential. The latter exhibits a linear dependency on the massless particle moving position on the light cone which in turn provides a constant and repulsive force $\approx v(c-v) / l$ and zero mass density in spacetime.) For case (2) the nonzero metric elements are simpler: $g_{00}=1-2 v t / l=1-2 x / l, g_{11}=-1-2 v^{3} t / c^{2} l=$ $-1-2 v^{2} x / c^{2} l, g_{11}=g_{11}=2 v^{2} t / l c$ and $g_{22}=g_{33}=-1$. And for fun again we can compare this metric with the Newtonian limit of GR to find a linear potential $\Phi=-x c^{2} / l$ but this time the constant repulsive force $\approx c^{2} / l$ is independent of the boost speed $v$, a familiar thing from dark energy dogma.

Before leaving this toy model let us outline briefly the possibility of altering equations (21a) and (21b) by replacing the second terms in the log functions by periodic functions which in turn lead to suggestive spacetime consequences not possible previously. In brief, we can set $x^{\prime}=(\gamma l c / v) \ln [1+$ $(v / c) \sin ((x-v t) / l)]$ and $t^{\prime}=(\gamma l / v) \ln [1+(v / c) \sin ((c t-$ $v x / c) / l$ )], which converge to the LT (modulo small corrections) for large $l$. A feature of curiosity now is specialized constant space and time translations $x \rightarrow x+x_{o}$ and $t \rightarrow$ $t+t_{o}$ could be performed in the $K$ system without ever affecting $x^{\prime}$ and $t^{\prime}$ in the $K^{\prime}$ system for the specific translations $x_{o}=c t_{o}=2 n \pi l c /(v(1-v / c))$. This intriguing possibility entails ample consequences that we leave out. Equally left out are the inverse transformations, velocity addition rule, quadratic forms, time dilation, and so forth. Regrettably these items must be omitted so to keep this paper reasonably short.

3.2. The NLT Core Proposal. The topic of nonlinear LT, especially at short distances not yet accessible, deserves more scrutiny and ought to be taken seriously in areas of Lorentz symmetry violations, and perhaps in par with the extension of the ordinary LT. The work conducted in this section, as already sampled in the earlier toy model and more to come shortly, reveals ample potentialities not foreseen in SR, including SL. No doubt there is room to include distinct upper speeds (be them subluminal or superluminal) in the fabric of the NLT, analogous to Section 2 for the case of linear LT. Definitely invoking NLT into physics impacts areas of relativistic QM, QFT, quantum gravity, the SM, the usual Lorentz invariant particle cross section, and so on. These topics are outside the limited scope of this paper but eventually must be included in the extended Lagrangian of any system. The major issue though is, unlike the LT and its defined group structure, that there is no unique formulation of the NLT in the absence of gravity, which is the scenery here where the nonlinear terms could be subjective in essence. Anyhow, to simplify life thereafter we consider the light speed $c$ as the only upper speed appearing in the NLT proposed hereafter. Moreover, for brevity sake no attempt is made to derive the involved energy-momentum dispersion relations to be treated elsewhere. Hereafter all NLT involve sums of linear and nonlinear terms, but the inverse NLT may not fit this menu.

We already saw an example of NLT in (21a) and (21b) and briefly discussed some of their key implications. But other forms are also possible. For example, set $t^{\prime}=\gamma\left[t-v x / c^{2}+\right.$ $\left.v t^{2} / c t_{o}\right], x^{\prime}=\gamma\left[x-v t+v t^{2} / t_{o}\right]$, where $t_{o}$ is a constant. The latter NLT respects the two-way light speed constancy when either the boost or the particle speeds reaches $\pm c$. Generally, the nonlinear terms violate the covariance of the physical laws in the sense of the SR principle postulated by Einstein. One peculiarity is the inverse NLT which is generally distinct in form from the NLT linking two IF in uniform relative motion. Enough has been said in the literature on diverse ways of realizing NLT that we skip over, but interested readers may check $[37,38]$ and the references therein. From our stand, hereafter we focus on only two phenomenological proposals forthcoming in two broad versions for exploring diverse nonlinear terms in the extension of the LT. We will engineer specific nonlinear terms purposely intended for massive elementary particles in Cartesian coordinates that would have dependencies on the rest masses and the Planck constant in their fabric.

Earlier we reminisced on one form of log/exponential type NLT which had an inbuilt sensitivity to an environmental (background) length scale $l$ that may be large or comparable to the particle probing region under study. Thereafter, we ditch the environmental Machian sway, as epitomized by the external length scale $l$, and strive in designing NLT where "everything" nonlinear occurs locally and at the same time keep an eye on a possible inclusion of a quantum length scale. In particular, we have a preference for a quantum length that can be associated with a boost related de Broglie inspired wavelength. Another issue of interest pursued here is the idea that the overall nonlinear term could be a solution of a wave equation of a sort. For example, an "energyless" wave skewed to an observer at rest in the stationary IF while appearing objective to an observer in the moving IF.

3.2.1. First Version. Inspired by the $\mathcal{O}(1 / l)$ limit of (21a) and (21b) (which are (23a) and (23b)) we now begin inferring the subsequent NLT:

$$
\begin{aligned}
& t^{\prime}=\gamma(v)\left[t-\frac{v x}{c^{2}}+(x-v t) \frac{F(x, t, v)}{c}\right], \\
& x^{\prime}=\gamma(v)\left[x-v t+\left(c t-\frac{v x}{c}\right) F(x, t, v)\right] .
\end{aligned}
$$


Here the boost $v$ is taken along the $x$-axis, $\gamma$ is as in SR, and $F$ is a real dimensionless scale function of $(x, t, v)$ in the $K$ IF (taken as the stationary frame). $F$ plays a major role in this version (the intriguing case of complex valued $F$ is dealt with elsewhere). The previous NLT, among other things, raise the déjà-vu possibility that a fixed observer in $K^{\prime}$ may not even become aware, depending on the right choice for $F$, of certain conditional (internal) space or time translations carried out in a few specified ways in $K$ (e.g., either $x \rightarrow x+a$ or $t \rightarrow t+b)$. Moreover, owing to the nonlinearity of such transformations (i.e., $F \neq 0$ ) there is the possibility of reviving the Galilean notion of simultaneity under certain stringent coordinate conditions. However, such exotic phenomena are not realizable for any choice of $F$, yet surely there exist functions that could yield explicit coordinate constraints making simultaneity possible. For example, an $F$ choice for making this possible in $K^{\prime}$, while also leading to sought constraint spacetime points $x(t)$, is $F(x, t, v)=(x-v t) / l_{o}$ where $l_{o}$ is some length scale. Consider two spatial points $x=0$ and $x$ at time $t$, and then require the resulting times in $K^{\prime}$ to be identical (thus simultaneity); that is, $t^{\prime}(x, t)=t^{\prime}(0, t)$. Simple algebra provides the sought spatial points in $K: x=v\left(2 t+l_{o} / c\right)$, satisfying $t^{\prime}(x, t)=t^{\prime}(0, t)$. The speed of a particle at such points is constant $w=d x / d t=2 v$, implying, in case $w \leq c$, then $v \leq c / 2$; so these mass points are only mildly relativistic and restricted to boost speeds lower than $c / 2$ and are also not accelerating in $K$. One can proceed further and determine the entire spacetime points in $K^{\prime}$ that are simultaneous, and the result is $t^{\prime}=\gamma t\left(1+2 v^{2} / c^{2}+4 v^{2} t / c l_{o}\right)$ and $x^{\prime}=\gamma v\left(t+l_{o} / c\right)[1-$ $\left.v^{2} / c^{2}+\left(c t / l_{o}\right)\left(1-2 v^{2} / c^{2}\right)\right]$. The case $l_{o} \gg 1$ (i.e., $F \gg 0$, where the NLT of (25) are approaching SR) is very special in that $w^{\prime} \approx v\left(1-2 v^{2} / c^{2}\right) /\left(1+2 v^{2} / c^{2}\right)$. Hence, for $v= \pm c / 2$ then $w^{\prime}=$ $\pm c / 6$. Of course these results are not general and only apply to the specific choice of $F$ made above; other plausible choices of $F$ may give very dissimilar results or no results at all.

Let us explore the NLT of (25) in more detail. It is easy to check $c t^{\prime} \pm x^{\prime}=A(v)(c t \pm x)(1 \pm F)$ where $A(v)=\sqrt{(1 \mp v / c) /(1 \pm v / c)}$. An upshot of this similar to the first toy model is that if the observer in the $K^{\prime}$ IF can perceive his spacetime as Minkowskian then inevitably from this observer's stand the $K$ IF is perceived as a convoluted Minkowski (conformally flat) space. Thus, at the level of spacetime quadratic intervals $S^{\prime}$ and $S$, defined usually in $M^{4}$, one finds $S^{\prime 2}=\eta_{\mu \nu} x^{\prime \mu} x^{\prime \nu}=c^{2} t^{\prime 2}-x^{\prime 2}=\left(c^{2} t^{2}-x^{2}\right)\left(1-F^{2}\right)=$ $\eta_{\mu \nu} x^{\mu} x^{\nu}\left(1-F^{2}\right)=S^{2}\left(1-F^{2}\right)$. As seen, the light cone remains invariant, another handy relation is $c^{2} t^{\prime} t-x^{\prime} x=\gamma\left(c^{2} t^{2}-\right.$ $\left.x^{2}\right)(1-F v / c)$. In addition, the relation $S^{\prime 2}=S^{2}\left(1-F^{2}\right)$ and assuming a Minkowski metric $\eta_{\mu \nu}$ in $K^{\prime}$ imply that the perceived metric $g_{\mu \nu}(x, t, v)$ in $K^{\prime}$ for the $K$ system is related to $\eta_{\mu \nu}$ by a conformal transformation $g_{\mu \nu}(x, t, v)=\phi(x, t, v) \eta_{\mu \nu}$ evaluated at a common point event viewed in the two distinct IF in uniform relative motion. The conformal factor $\phi(x, t, v)=1-F^{2}(x, t, v)$ causes a local change of scale, stretching or shrinking distances in $M^{4}$. (In GR a conformal factor contributes three extra terms to the original affine connection proportional to the first spacetime derivatives of $\ln (\phi)$, and forcibly affecting the original geodesics.)
The nonvanishing Lorentz tensor elements $\Lambda_{\mu \nu}(x, t, v)$, linking $(t, x, y, z)$ to $\left(t^{\prime}, x^{\prime}, y^{\prime}, z^{\prime}\right)$, with the boost along $x$, are $\Lambda_{00}=\Lambda_{11}=\gamma(1-F(x, t, v) v / c), \Lambda_{22}=\Lambda_{33}=1$, $\Lambda_{01}=\Lambda_{10}=\gamma(F-v / c), \Delta=\operatorname{det}(\Lambda)=1-F^{2}$. For the inverse nonlinear transformations the nonzero Lorentz tensor elements are $\Lambda_{00}^{-1}=\left(\Lambda^{-1}\right)_{11}=\Lambda^{-1} \gamma(1-F(x, t, v) v / c)$, and $\Lambda_{22}^{-1}=\Lambda_{33}^{-1}=1, \Lambda_{01}^{-1}=\Lambda_{10}^{-1}=-\Lambda^{-1} \gamma(F-v / c)$, where the inverse determinant is $\Lambda^{-1}=\operatorname{det}\left(\Lambda^{-1}\right)=\left(1-F^{2}\right)^{-1}$. The inverse NLT differ in form from the NLT and are $t=$ $\Lambda^{-1} \gamma(v)\left[t^{\prime}+v x^{\prime} / c^{2}-\left(x^{\prime}+v t^{\prime}\right) F / c\right]$ and $x=\Lambda^{-1} \gamma(v)\left[x^{\prime}+v t^{\prime}-\right.$ $\left.\left(c t^{\prime}+v x^{\prime} / c\right) F\right]$. For small $F$, more concisely when $F^{2}$ can be safely neglected (i.e., $\Lambda \sim 1$ ), one (more or less) recovers the invariance of these NLT equations as in SR. But when $F$ is not small the inverse transformations, and thus the anisotropy, are not form preserving, except at spacetime points whereby $F=0$ (if existent).

The previous findings were at the level of spacetime, but at the manifold level the central character is evidently the metric. The line element square in $K^{\prime}$ is set as $\left(d S^{\prime}\right)^{2}=c^{2} d t^{\prime 2}-$ $d x^{\prime 2}-d y^{\prime 2}-d z^{\prime 2}$. But when $\left(d S^{\prime}\right)^{2}$ is expressed in the $(x, t)$ coordinates it reads $c^{2} d t^{\prime 2}-d x^{\prime 2}=d\left(c t^{\prime}+x^{\prime}\right) d\left(c t^{\prime}-x^{\prime}\right)=$ $d[(1+F)(c t+x)] d[(1-F)(c t-x)]$. Specific forms of $F$ can be now sought so as to force (if possible) the quadratic line element to take on the reduced form: $c^{2} d t^{\prime 2}-d x^{\prime 2}=$ $\left(c^{2} d t^{2}-d x^{2}\right)\left(1-F^{2}\right)$ mimicking the interval link in the previous paragraph where the two metrics were related by a conformal transformation. Skipping details, the candidate $F$ must be obtained by solving a rather involved differential equation: $F=d\left[\ln \left(e^{-F}(c t-x) /(c t+x)\right)\right] / d(\ln I)$, where $I=c^{2} t^{2}-x^{2}$.

Note. From the previous quadratic phenomenology just talked over one could draw an analogy with the one existing between the Riemannian geometry and the Finsler geometry where the latter is viewed as a Riemannian geometry without quadratic restriction. In the F-geometry, Lorentz invariance violation stems from the particular Finslerian metric $g_{\mu \nu}(x, d x)$, so that $d S^{2}=g_{\mu \nu}(x, d x) d x^{\mu} d x^{\nu}$ involves, besides the coordinates $x$, also the local speeds $d x^{\mu} / d \tau$.

It is imperative that the quadratic intervals $S^{\prime 2}$ and $S^{2}$ have the same spacetime signatures, meaning $F^{2} \leq 1$. Or else for a selected $F$ in $K$ one may find certain time-like events $\left(S^{2}>0\right)$ such that $F^{2}>1$ at those points, and then in $K$ the observer reads $S^{\prime 2}<0$, thus violating causality. Such tachyon-like events in $K^{\prime}$ may be pertinent to superluminal signals at those points. The goal at the end of this version is to include a vestige of QM in $F$ designed to have many spacetime zero points, whereby the invariance of the linear LT, the interval and the line element are recovered exactly at such points. At the same time, $F$ ought to decrease rapidly to zero at all other points beyond few separation lengths measured by a "global" de Broglie look-alike wavelength defined as $\lambda=\hbar / m v$, where $v$ is the boost speed. (It is reminded that the least quantities that two inertial observers can always agree on, besides the fundamental constants, are the rest mass $m$ and the fixed boost speed $v$ between the two IFs. So a quantity like $\lambda=\hbar / m v$ (and of course $\hbar / m c$ ) reads the same by either inertial observers or 
coordinate systems.) Despite the NLT of (25), and the clock synchronization synchronized at $t^{\prime}=t=0$ and $x^{\prime}=x=0$, both reference frames begin as customary Minkowskian at $v=0$, but it is because of the boost $v \neq 0$ and the NLT that the $K^{\prime}$ observer perceives the $K$ space as deformed Minkowskian, and vice versa replacing $F(x, t, v) \rightarrow F\left(x^{\prime}, t^{\prime},-v\right)$. (Had this been GR then for $F \ll 1$ the additional metric $-\eta_{\mu \nu} F^{2}=h_{\mu \nu}$ could be interpreted as a small perturbation to flat-space metric giving rise to linearized GR and the phenomenon of gravitational waves where $\square h_{\mu \nu}=0$ and upon quantization spin 2 gravitons ensue.) Evidently, to recover the usual LT one must have a vanishing $F$ at both $v=0$ and at all specific spacetime points where $F=0$; then for any $v$ the standard LT applies at such points. We will choose from then on the option $F(x, t, v)=\epsilon(v) f(x, t)$. A Plausible candidate, though not unique, for $\epsilon(v)$ is $\sim\left(1-v^{2} / c^{2}\right)(v / c)^{\alpha}$ with $\alpha>0$.

There is no inclusive menu for constructing $f(x, t)$, but in simplest of cases one could try a polynomial, trigonometric, hyperbolic, or sinc functions in $x$ and $t$. Soon we will provide an expression for $f$ and examine it in some detail. But even before designing $f$ certain early information on the behavior of $F$ can be obtained through defining average particle speeds along $x$ in $K$ and $K^{\prime}$. Define $\bar{u}=x / t$ and $\bar{u}^{\prime}=x^{\prime} / t^{\prime}$, and then plug these into (25) to get $\bar{u}^{\prime}=\left[\bar{u}-v+F c\left(1-v \bar{u} / c^{2}\right) /(1-\right.$ $\left.\bar{u} v / c^{2}+(\bar{u}-v) F / c\right]$. Now, in the limit $v=0$ we expect $\bar{u}^{\prime}=u$ so that $F(x, t, 0)=0$; moreover, for $v= \pm c$ we set $F(x, t, v=$ $\pm c)=0$ leading to $\bar{u}^{\prime}=\mp c$. It is also easy to show that for all $F(x, t, v)$ then $\bar{u}= \pm c \rightarrow \bar{u}^{\prime}= \pm c$. Hence, the constancy of the light speed is achieved in both IF as in SR. Moreover, when $\bar{u}=v$ (which is zero in SR) one finds $\bar{u}^{\prime}(\bar{u}=v)=$ $c F(x=v t, t, v)$. This shows that $F(x=v t, t, v)$ must be fairly small, if not zero, in most speed ranges $v<c$, and in case $v=c$ then $\bar{u}^{\prime}(\bar{u}=v=c)=0$ as in SR. Hence, an expression like $\epsilon(v) \sim(v / c)^{\alpha}\left(1-v^{2} / c^{2}\right)$ is both sensible and appropriate. We can also derive the local velocity addition rule directly from (25), as it is done later.

The exact velocity addition rule is $u^{\prime}(x, t, u, v)=[u(1-$ $F v / c)-v+c F+(c t-v x / c) \dot{F}] /\left[1-u v / c^{2}+(u-v) F / c+(x-v t) \dot{F} / c\right]$ where $\dot{F}=d F / d t$. Adopting the form $F(x, t, v)=\epsilon(v) f(x, t)$, with $\epsilon( \pm c)=0$, leads to the constancy of the light speed in inertial frames in relative uniform motion, meaning for the boost $v= \pm c$ then $u^{\prime}=\mp c$. Likewise, in case $u= \pm c$ then $u^{\prime}= \pm c$ for any $v$ and $F$. For the special case $u=v$ then in general the particle worldline satisfies $x=v t+x_{o}$. In this case, $u^{\prime}=c\left[F+\left(t-v x_{o} /\left(c^{2}-v^{2}\right)\right) \dot{F}\right] /\left[1+x_{o} c \dot{F} /\left(c^{2}-v^{2}\right)\right]$, and a suitable tryout form $f\left(x=v t+x_{o}, t\right)=\left[1-\left(c^{2}-v^{2}\right) t / v x_{o}\right]^{-1}$ is able to give the desirable result $u^{\prime}=0$ which is also identical to the result expected in SR.

A task remaining is consequently seeking novel models for $f(x, t)$, in particular those periodic in coordinates. The way we attempt that this is inspired by the trivial observation that both in SR and the Galilean relativity the coordinates $\left(x^{\prime}, t^{\prime}\right)$ or $(x, t)$ obey a homogeneous wave equation $\square_{s}\left(x^{\prime}, t^{\prime}\right)=0$ or $\square_{s}(x, t)=0$, where $s$ is the wave arbitrary propagation speed. This "math" triviality is of little physical undertone in SR, where gravity is absence, but becomes a useful and a simplifying tool in 4D GR where it is possible to define "harmonic" coordinates in curved space satisfying $\square_{c} x^{\mu}=0$. Harmonic coordinates are also widely used in the weak field limits. But for the NLT of (25) things are more cumbersome. If in the context of a single function $f$, defining the NLT, one insists on satisfying both $\square_{s} x^{\prime}=0$ and $\square_{s} t^{\prime}=0$ simultaneously (i.e., $\square_{s}[f \cdot(x-v t)]=0$ and $\square_{s}[f$. $(c t-v x / c)]=0$ concurrently), then certain unwanted intricacies arise unless not one but two distinct functions $f(x, t)$ and $g(x, t)$ are included in the design of the NLT.

As long as a single function $f$ is used to define the NLT we find it doubtful to satisfy the previous two $1 \mathrm{D}$ wave equations at once under a common propagation speed $s$. Yet, this can be realized if instead two constant propagation speeds $\tilde{u}$ and $s$ are invoked. Here is a generic example. Set $f=a_{o}[x-\widehat{c} t(\widehat{c} / c)]$, where $\widehat{c}$ is an arbitrary speed, and $a_{o}$ is a constant having an inverse length unit. Then $\square_{\tilde{u}}[f \cdot(x-v t)]=0\left(\rightarrow \square_{\tilde{u}} t^{\prime}=0\right)$ is satisfied if $\widetilde{u}^{2}=\hat{c}^{2}(v / c)$, and likewise $\square_{s}[f \cdot(c t-v x / c)]=0$ (so $\square_{s} x^{\prime}=0$ ) if $s^{2}=\widehat{c}^{2}(c / v)$. In particular, for $\tilde{u}=v$ (the boost speed) then $s=c$ which is an interesting result in need of elucidation. However, in case $\widehat{c}=c$ then $\widetilde{u}^{2}=$ $c v<c^{2}$ and $s^{2}=c^{3} / v \geq c^{2}$. Implying the wave propagation speed $s$ in the spatial dimension is superluminal, and this is acceptable since no energy is transmitted. Such issues and the likes often occur also for other generic examples when choosing spacetime polynomials for $f$ that we prefer to avoid. Conversely chosen aperiodic polynomials for $f$ are also not too interesting from the physics stand; but things become more rousing when periodic waves are invoked in the making of the $f$ function. Visibly, no reference is made here on $f$ emitting real and dispersionless waves carrying energy. There is no contemplation of gravitational waves or E\&M radiation here when allowing for $f$. All there is are idealized massive particles moving in $M^{4}$, fairly analogous to minimal GR in curving spacetime.

Note. We are aware that there can be a conceptual issue in regards to our wave-nonlinear coordinate duality conjecture as to whether it should apply in the absence of a massive particle observation of a sort involving two macroscopic IFs in uniform relative motion. This is a conjecture demarcating it from GR and noninertial frame intricacies from the onset. Such a wave-nonlinear coordinate duality may have a similarity in spirit with the quantum mechanical perceptive and even more so when $f$ is treated as a small perturbation in the NLT. No doubt $f$ must act as small corrections to the LT, be its source classical or quantum mechanical. In the absence of real physical field sources (like gravity, E\&M, etc.) to cause nonlinearities and vice versa (a topic quite apart from what we are trying to promote here) then the do-it-yourself waves are subjective and are solely due to small nonlinear parts encoded in the LT and only exist while a boost is carried out. So in a sense this opens up the possibility of bringing aboard not the full but a dash of boost related QM corrections of a sort to feature the nonlinear terms. One way to do that is to directly encode the Planck constant (which is send to zero for macroscopic objects) and possibly the rest mass, in the nonlinear term fabric, while leaving behind the rest of the QM formalism. Said differently, since "no boost = no $f=$ no waves," then nonlinearity is not an intrinsic property of spacetime itself (at the level of SR) but comes to perception 
by one IF observer only upon motion. Implying the wavenonlinearity is not related to the complex quantum state function $\Psi=R e^{i S}$, the Hilbert space, and so forth. The subsistence of $\Psi$ and the overall uncertainty principle in a given frame should have nothing to do with the NLT acting between the IFs. (Yet we must confess seeing in the earlier interval relation $S^{\prime 2}=S^{2}\left(1-F^{2}\right)$ a spark of appeal for the possibility of interpreting the conformal factor $\left(1-F^{2}\right)$, perceived by $K^{\prime}$, as a classical probability of finding a point particle at $(x, t)$ in the $K \mathrm{IF}$, so that the term $F^{2}$ can stand for the probability that a particle is not at $x$ at time $t$ in $K$. In brief, when the classical particle is "there" the LT are linear at "there" and $F^{2}=0$, and when not probed at "there" then $F^{2} \neq 0$. Even though it is not obvious how far this idea can stretch we think it deserves further examination.

Although nonlinear LT are permitted here to act between two uniformly moving classical IF in $M^{4}$, the massive point events could themselves be accelerating in these inertial frames through the quantum vacuum of the Minkowski space serving as the background space. Meaning, the accelerating point masses in say the IF $K$ could experience a thermal bath and thermal distribution at the well-known Unruh temperature proportional to their accelerations and the reduced Planck constant. In what follows we will have to disregard such quantum effects so to prevent having Planck type correlation functions for accelerating particles in $M^{4}$. Besides, it is unlikely the Unruh phenomenon can be the source of nonlinearities in the NLT, and therefore it is ignored.

Next, we discard polynomial forms of nonlinearities in the NLT and turn instead to periodic functions by proposing a variant to (25) featuring some wavy quantum attributes and also making it possible to set $\square_{s} x^{\prime}=0$ and $\square_{s} t^{\prime}=0$ under a common generic propagation speed $s$. The nonlinear and generic term is set initially as $F(x, t, v)=\epsilon(v)[(A x+$ $B \tilde{u} t+k) \sin (k x) \cos (k \tilde{u} t)+\cos (k x) \sin (k \tilde{u} t)(B x+A \tilde{u} t+k)]$ where $k=2 \pi / \lambda$ with $\lambda=h / m v$ (note that $\lambda \nu=v$, where $v$ is the wave frequency), $A$ and $B$ are constants, $v$ is the boost speed, $m$ is the rest mass, and $\tilde{u}$ is some constant speed scale. It is now trivial to show that $\square_{\widetilde{u}} F=0$. But to prevent the growth of nonlinearities in the NLT with time and space we set $A=B=0$. It is of course possible to seek a broader expression for $F$ by considering, for example, a Fourier series for $F$ in terms of periodic functions in $(x, t)$, but for brevity sake we use only a single sine function to depict the nonlinear term in the NLT. The new NLT variants, also realizing the constraints $\square_{\widetilde{u}} x^{\prime}=0$ and $\square_{\widetilde{u}} t^{\prime}=0$, are

$$
\begin{gathered}
t^{\prime}=\gamma(v)\left[t-\frac{v x}{c^{2}}+\frac{F(x, t, v)}{2 k c}\right], \\
x^{\prime}=\gamma\left[x-v t+\frac{F(x, t, v)}{2 k}\right] .
\end{gathered}
$$

Here, $k=2 \pi / \lambda, F(x, t, v)=\epsilon(v)(1-v / c) \sin (k(x+\tilde{u} t))$, and $\epsilon(v)=\epsilon_{o}\left(1-v^{2} / c^{2}\right)(v / c)^{\alpha}$, with $0<\epsilon_{o}<1$. The speed addition law along $x$ axis is $u^{\prime}=\left[(u-v+\dot{F} / 2) /\left(1-u v / c^{2}+\dot{F} / 2\right]\right.$ with $\dot{F}=k \epsilon(v)(1-v / c)(u+\tilde{u}) \cos (k(x+\tilde{u} t)$. For $v= \pm c$ then $u^{\prime}=\mp c$, for all $u$, as in SR. For a particle moving at $u= \pm c$ (and setting $x= \pm c t$ ) slight time dependent periodic deviation from light speed is observed for $u^{\prime}$ (unless the wavy term propagates with speed in the opposite direction: $\tilde{u}=$ $\mp c$ ). Of particular interest is the case when the particle is at rest in $K^{\prime}$. In this case predictions for $u^{\prime}$ differ from SR (which is zero) where $u^{\prime}$ is $(k / 2) \epsilon(v)(1-v / c) \cos (k(v+\widetilde{u}) t$ divided by a nonzero denominator. This finding may have an import from the physics stand. At this point because of the $(x, t)$ dependency of $u^{\prime}$ it is hard to tell whether superluminal speeds could result; so it is left as an open question. Finally, the inverse NLT transformations of (26) are too involved to be shown here.

We now explore the key quadratic forms for (26). The first quadratic form is the interval link given as

$$
c^{2} t^{\prime 2}-x^{\prime 2}=\left(c^{2} t^{2}-x^{2}\right)\left[1-\epsilon(v) \frac{\sin (k(x+\tilde{u} t))}{k(x+c t)}\right] .
$$

Equation (27) implies for wave speed $\tilde{u}=c$ the conformal factor simplifies to a desirable sinc function in $x+c t$. Note also that both the usual linear LT and the Minkowski metric in $M^{4}$ are restored at all spacetime events satisfying the discrete constraint $x+\tilde{u} t=(n / 2) \lambda$ where $n$ is an integer and $\lambda=h / m v$. In general, the conformal factor in (27) converges rapidly to unity for all spacetime points $(x+c t)>4 \lambda$, or so. The next query is at the manifold level involving the line element:

$$
\begin{aligned}
& c^{2} d t^{\prime 2}-d x^{\prime 2} \\
& \quad=\left(c^{2} d t^{2}-d x^{2}\right)\left[1+\epsilon(v) \cos (k(x+\tilde{u} t)) \frac{u+\widetilde{u}}{u+c}\right] .
\end{aligned}
$$

Here $u$ is the particle instantaneous speed along $x, u=$ $d x / d t$, and for $\tilde{u}=c: d S^{\prime 2}=d S^{2}[1+\epsilon(v) \cos (k(x+$ $c t))]$. Finally, it is important noticing the marked difference between the perceived conformal factors gotten from (27), via the interval connection, and the one just presented via the line element in (28).

3.2.2. Second Version. We conclude this paper by presenting the second and last version of our NLT study which, as already stressed, differs from those discussed in the first version (i.e., (25) and (26)) in that the single nonlinear term $F$ appearing in the first version NLT is now extended to two distinct functions. The broad makeup of the new NLT is $t^{\prime}=\gamma(v)[t-$ $\left.v x / c^{2}+\epsilon_{1}(v) f(x, t, v)\right]$ and $x^{\prime}=\gamma(v)\left[x-v t+\epsilon_{2}(v) g(x, t, v)\right]$. To constraint the two functions $f$ and $g$ we require the nonlinear correction term induced in the quadratic interval be a perfect square and contain no terms proportional to $f$ and $g$. As the result $f$ and $g$ become related: $\epsilon_{2} g(c t-v x / c)=\epsilon_{1} c f(x-v t)$. Now, by setting $\epsilon_{1}=\epsilon_{2}=\epsilon$ the interval relation reads $c^{2} t^{\prime 2}-x^{\prime 2}=\left(c^{2} t^{2}-x^{2}\right)\left(1-Q^{2}\right)$ where $Q^{2}=\epsilon^{2} g^{2} /(x-v t)^{2}$. Of course there is no unique way of choosing the $g$ function, and all we can do is to try a number of reasonable forms for $g(x, t, v)$. Earlier we expressed the desire to include periodic functions, and in particular the sinc functions, in the NLT. Having a sinc function in the interval connection causes a rapid decrease of $Q^{2}$ beyond some length scale (preferably of quantum origin already alluded to in the first scenario), thus restoring the usual quadratic interval of SR quickly. We see from the expression of $Q^{2}$ the simplest choice to get a sinc 
function is to set $g=\sin (k(x-v t)) / k$, where $k$ (see later) has a unit (length) ${ }^{-1}$.

Earlier we discussed reasons for setting $k=2 \pi / \lambda=$ $m v / \hbar$ with $v$ the boost speed. This choice is reasonable, and it remains the same for two inertial observers in relative uniform motion while imparting also a dash of QM and the rest mass to only the nonlinear terms in the NLT. And, moreover, it does not change the Minkowski spacetime to a kind of quantum spacetime, per se, and finally $k$ also vanishes when $v \rightarrow 0$. Yet, there may be a serious objection for this pick as to why take an arbitrary quantum particle mass under probing and then collocate to it the entire spacetime in the NLT, involving uncountable geometrical points in either inertial frame, mostly never encountering a mass in motion normally located along its worldline. Besides, many real particles can decay rapidly, then what to make of that with such NLT? The answer is not simple, but the key word is "quantum particle." A quantum particle has a wavefunction spanning spacetime and is also an abstract probabilistic notion (though maybe not to Bohm and his follower's view) allowing particles to be everywhere at once, but so is the notion of geometrical points in spacetime devoid of any physical emblem (recall the operational meaning of vacuum in QFT). It is therefore not fortuitous that we have chosen the previous specific expression for $k$ to simply avoid having any local definition for it, while knowing this choice makes no reference to spacetime points, or even the momentum space points, yet it still personalizes the tiny nonlinear terms in the NLT to a specific particle of mass $m$. whether this is good or bad we cannot tell, though we are aware that including particle masses in the NLT can cause a mixing of kinematics (when deriving acceleration) and dynamics through the relativistic force $F^{\mu}=m d u^{\mu} / d \tau$. If the nonlinear terms are to be universal then this objection can be removed by replacing the renormalized mass $m$ by a GUT mass scale, or better the Planck mass invoking the universal gravitational constant $G$.

Anyhow, the proposed NLT for this last version and its resulting interval connection shown in (31) $\left(\epsilon(v)=\epsilon_{o}(1-\right.$ $\left.v^{2} / c^{2}\right)(v / c)^{\alpha}$ is constantly a small quantity, and $\epsilon(0)=\epsilon(c)=$ $0)$, are

$$
\begin{gathered}
t^{\prime}=\gamma\left[t-\frac{v x}{c^{2}}+\epsilon(v) \frac{\sin (k(x-v t))}{k c}\right], \\
x^{\prime}=\gamma\left[x-v t+\epsilon(v)\left(c t-\frac{v x}{c}\right) \operatorname{sinc}(k(x-v t))\right], \\
S^{\prime 2}=S^{2}\left(1-\epsilon(v)^{2} \operatorname{sinc}^{2}(k(x-v t))\right) .
\end{gathered}
$$

The sinc function in the spatial transformation $x^{\prime}$ and $S^{\prime 2}$ eventually disappears beyond some length scale restoring the familiar SR expressions, while the sine function in $t^{\prime}$ persists as a small fluctuation in the time transformation. But there is more, as the linear and the nonlinear terms can conspire to force (29) and (30) to become linear relations at specific points satisfying the discrete condition $x-v t=n \pi / k$, so that $x^{\prime}=\gamma n \pi / k$ and $t^{\prime}=t / \gamma-\gamma n \pi v / k c^{2}$. When integer $n$ could change in time deterministically or stochastically it could have a deep physics nuance that we pass over. Finally, It is easily checked that for any point $(x, t): \square_{v} t^{\prime}=0$, but $\square_{v} x^{\prime} \neq 0$; so its associated wave equation is inhomogeneous and has a source term. The line element ensuing from (29) and (30) is too complex to outline here and is omitted.

Let us now turn briefly to some features of the new velocity addition rule for $u^{\prime}$ obtained from (29) and (30). It is expectedly a long expression involving the usual SR term $(u-v) /\left(1-u v / c^{2}\right)$ multiplied by a corrective factor involving sine and cosine functions. We will not provide the full lengthy expression for $u^{\prime}$. Suffice to say that the constancy of the light speed is a consequence of these NLT in the following sense. When $v= \pm c$ then $u^{\prime}=\mp c$, for all $u$, and this is exactly as in SR. When a particle speed is $u=c$ (and setting $x=c t$ ) then $u^{\prime}=c$, for all $v$, and likewise for $u=-c$ (setting $x=-c t$ ) then $u^{\prime}=-c$, for all $v$. We have deliberately omitted here the most general solution points for $u= \pm c$ which are $x= \pm c t+x_{0}$, involving the initial positions $x_{0}$. The initial positions cause an asymmetry in $u^{\prime}$ (albeit tiny) when the particle speed reaches $u= \pm c$.

The algebraically closed inverse transformations of (29) and (30) are complicated but become easier to handle for the case when $k(x-v t)$ is a small perturbation. We will not present them here. But there is a relation one can get by eliminating the sine functions from (29) and (30) to obtain the suggestive hybrid interval relation: $c^{2} t^{2}-x^{2}=\gamma\left[c^{2} t^{\prime} t-x^{\prime} x+v\left(x^{\prime} t-\right.\right.$ $\left.\left.x t^{\prime}\right)\right]$. This equation, in turn, can serve to check simultaneity. Let us entertain this possibility as an exercise. Imagine an event occurred at $(x, t)$ in $K$. Suppose that this event can be observed in $K^{\prime}$ at some $x^{\prime}$ simultaneously (i.e., $t^{\prime}=t$ ).

(1) Find $x^{\prime}$. Solution: $x^{\prime}=(x-v t)^{-1}\left[c^{2} t^{2}-x v t-\gamma^{-1}\left(c^{2} t^{2}-\right.\right.$ $\left.x^{2}\right)$.

(2) By substituting $x^{\prime}$ derived in (1) into (30) find the relationship between the candidate points $x$ and $t$ in K. Solution: $\epsilon(v)(c t-v x / c) \sin (k(x-v t)) / k=\left(\gamma^{-1}-\right.$ 1) $\left(c^{2} t^{2}-2 x v t\right)-v^{2} x^{2} / c^{2}$

(3) Evaluate (2) in the limit $v=c$. Solution: $x=c t$, which is the expected result.

A topic never talked about before is the link between the spatial accelerations $a$ and $a^{\prime}$ of massive particles as observed in two inertial frames $K$ and $K^{\prime}$ in constant relative motion along $x$. Standard SR provides the relation $a^{\prime}=a\left[\gamma\left(1-u v / c^{2}\right]^{-3}\right.$ where even for the extreme case $u=c$ does not indicate a finite maximal acceleration. (Even for the combined case $v=c$ and $u=c$ one still finds $a^{\prime} \rightarrow \infty$.) So the issue now is whether some of the extended linear or nonlinear LT discussed in this work could provide a hint of a maximal acceleration. It seems doubtful that this can happen for the linear LT extensions proposed in Section 2. Yet for the NLT discussed in this section, one cannot rule out this possibility. Computational complexities, however, prevent us from reaching any definite conclusion; so the issue of a maximal acceleration in the context of our NLT remains an open topic for inquiry. Finally, once it is conjectured that Lorentz symmetry may be (or should be) violated (as contemplated by effective string theory and a number of pointlike field theories) then involving the NLT into the query may be the next natural step to take for enlarging SR and 
perhaps for investigating SL via the extra analytic tools they can provide not foreseeable in SR.

To conclude, from the start we adhered to the trivial norm practiced in experimental physics that even if all the available data on neutrinos, or in general any other known particle $X$, are compatible with subluminal speeds most existing data uncertainties are not at the level that a tiny superluminality can be precluded outright. More specifically, as long as the ratio $\delta_{X}=\left(v_{X}-c_{\gamma}\right) / c_{\gamma}$ for a particle $\mathrm{X}$ admits a positive value, within the data uncertainties, speculating about SL (like for the neutrinos) is a legitimate option. In Section 2, a plethora of proposals was contextualized in form of two independent scenarios pertaining to extended linear LT, all of which were fabricated to specifically cover superluminality. In the first scenario while the usual LT were formally kept intact, the hub was to replace in the LT $v$ by $v \times f(v)$ (see (3)-(5)) where $v \times f(v) \leq c$ for any $v$, even in the superluminal range $v>c$ as allowed by a particular choice of a suitable $f(v)$. The core of this substitution is even if two inertial observers may agree unambiguously on $v$ as the global boost speed magnitude between two IFs in uniform relative motion, what goes in the making of the modified LT, pertaining to local spacetime transformations of "physical" point particles, and the ensuing kinematics, is not the global boost speed $v$ but rather $v \times f(v)$, where $f(v)$ is a continuous function of $v$ with $0 \leq f(v) \leq 1$. As a result of this substitution it is shown towards the end of Section 2.1 complicated alterations of the $E(P)$ dispersion relations are inevitable. Consistency at the Galilean limit $v \ll$ $c$, and at the exact $v=c$ limit, requires $f(0)=f(c)=1$. Thus, as in the standard LT here too when $v=c, \gamma \rightarrow \infty$. The main challenge in the first scenario is to model $f(v)$, and therefore the modified $\gamma$ factor. Only four realizations of the $\gamma$ factor, among other possible options (like considering slowly varying $\log$ functions), were discussed in the text and also illustrated in Figure 1. As seen, the fourth one, though not perfect, is the finest. The ideal model of $f(v)$ is the one that produces a $\gamma$ factor for massive particles exceedingly close, but not necessarily equal, to the usual $\gamma$ factor of SR in all relativistic subluminal regions $v \leq c$ (i.e., in the ideal case the difference between both $\gamma$ factors is such that for any $v<c$ the observed particle energy-momentum is within the experimental uncertainties). An attempt was also made in this scenario to elucidate the physical origin of $f(v)$, but as of this writing the exact reason for its existence is still lacking. The second scenario in Section 2 pertains to modified LT endowed with two rest mass dependent superluminal speeds (see (9), (10) and ((17a), (17b), and (17c))). This scenario was utilized to evaluate the energy of a host of particles (including the neutrinos) at the exact $v=c$ limit, where the $\gamma$ factor is finite. The latter evaluation was shown to be compatible with a host of ultra-relativistic high energy cosmic ray data. Overall, the velocity composition rules in this scenario (13) reveal a high degree of consistency in the two-way light speed constancy, yet for certain upper speed limits (see items 16) it also allows for tiny speed asymmetries when viewed in two distinct IF in uniform relative motion. In Section 3 a variety of novel looking nonlinear LT (NLT) were proposed with many inspiring technical up shots which we rather leave out elaborating here. In that section for simplicity sake the light speed is incorporated in all NLT models to stand as the only fundamental upper speed input. Here too an overall compatibility with the light speed constancy is observed for most NLT. Certain hints of simultaneity for a limited set of spacetime events, and, independently, hints of superluminality, were also found in few of the NLT models discussed in Section 3. In general though, because of technical subtleties in such models the investigation of whether under a subluminal boost certain specific local spacetime points in one chosen IF could appear superluminal in the other IF in uniform relative motion is not a trivial task. Yet, as it was done in Section 2 , it is always possible to invoke from the start SL upper speed(s) in the design of the NLT; this could be an interesting project of inquiry for the future. There is little doubt that in any physical framework-be it quantum mechanical or semi classical, stringy or point like, involving SL or not-where Lorentz invariance violations (LIV) may exist, or anticipated, reasonably well designed NLT could be a powerful tool for investigating LIV. Our limited practice in designing NLT in Section 3 has convinced us that in the absence of gravity (i.e., GR) nonlinear LT, including SL or not, could have distinctive theoretical potentialities and advantages over the extended LT. But since the devil is in the detail, more study is needed on a large number of NLT, and even more so given that a formal group structure for such transformations, in contrast to most linear LT, may not always exist, to assess their merits and challenges in particle physics and QFT. The physics community is encouraged to pay more attention to this often disused prospect of relativistic physics; one can never tell of unexpected surprises that could show up in studying NLT.

\section{References}

[1] M. Longo, “Tests of relativity from SN1987a," Physical Review D, vol. 36, no. 10, pp. 3276-3277, 1987.

[2] The OPERA Collaboration, T. Adam, N. Agafonova, A. Aleksandrov et al., "Measurement of the neutrino velocity with the OPERA detector in the CNGS beam," http://arxiv.org/ abs/1109.4897v1. In press.

[3] The OPERA Collaboration, T. Adam, N. Agafonova, A. Aleksandrov et al., "Measurement of the neutrino velocity with the OPERA detector in the CNGS beam," http://arxiv.org/abs/ 1109.4897. In press.

[4] The OPERA Collaboration, T. Adam, N. Agafonova, A. Aleksandrov et al., "Measurement of the neutrino velocity with the OPERA detector in the CNGS beam using the 2012 dedicated data," http://arxiv.org/abs/1212.1276. In press.

[5] The MINOS Collaboration, P. Adamson, C. Backhouse, G. Barr et al., "Measurement of neutrino velocity with the MINOS detectors and NuMI neutrino beam," http://arxiv.org/ abs/0706.0437. In press.

[6] MINOS collaboration, P. Adamson, D. S. Ayres, G. Barr et al., "Search for Lorentz invariance and CPT violation with muon antineutrinos in the MINOS Near Detector," http://arxiv.org/ abs/1201.2631. In press.

[7] ICARUS Collaboration, M. Antonello, P. Aprili, B. Baibussinov et al., "A search for the analogue to Cherenkov radiation by high energy neutrinos at superluminal speeds in ICARUS," http://arxiv.org/abs/1110.3763. In press. 
[8] M. Antonello, P. Aprili, B. Baibussinov et al., "Measurement of the neutrino velocity with the ICARUS detector at the CNGS beam," http://arxiv.org/abs/1203.3433. In press.

[9] K. Peacock, "Would superluminal influences violate the principle of relativity?" http://arxiv.org/abs/1301.0307. In press.

[10] J. Hill and B. Cox, "Einstein's special relativity beyond the speed of light," Proceedings of the Royal Society A, vol. 468, no. 2148, pp. 4174-4192, 2012.

[11] G. Ghirardi and R. Romano, "On a proposal of superluminal communication," http://arxiv.org/abs/1205.1416. In press.

[12] K. Fujii, "Superluminal group velocity of neutrinos: review, development and problems," http://arxiv.org/abs/1203.6425. In press.

[13] I. Oda, "Subluminal OPERA neutrinos," http://arxiv.org/abs/ 1112.5793. In press.

[14] G. Ter-Kazarian, "Extended Lorentz code of a superluminal particle," http://arxiv.org/abs/1202.0469. In press.

[15] Z.-Y. Wang, C.-D. Xiong, and B. He, "Superluminal propagation of evanescent modes as a quantum effect," http://arxiv.org/ abs/0712.0347. In press.

[16] A. Y. Li, "Relativistic theory of superluminal particles," http:// arxiv.org/abs/1110.0969. In press.

[17] V. Gupta, "A modification of the relativistic energy-momentum relation," http://arxiv.org/abs/1204.4478. In press.

[18] J. Schechter and M. N. Shahid, "Neutrinos with velocities greater than $c$ ?" http://arxiv.org/abs/1201.4374. In press.

[19] H. Behera and G. Mukhopadhyay, "On the invariance of the speed of light," http://arxiv.org/abs/1111.6516. In press.

[20] G. Guo and X. -G. He, "Dispersion relations explaining OPERA data from deformed lorentz transformation," http://arxiv.org/ abs/1111.6330. In press.

[21] M. E. Perel'man, "Uncertainty relations describing the shortterm violations of Lorentz invariance: superluminal phenomena, particles transformations," http://arxiv.org/abs/quant-ph/ 0510123. In press.

[22] L. Gonzalez-Mestres, "Lorentz invariance and superluminal particles," http://arxiv.org/abs/physics/9703020. In press.

[23] G. Amelino-Camelia, G. Gubitosi, N. Loret, F. Mercati, G. Rosati, and P. Lipari, "OPERA-reassessing data on the energy dependence of the speed of neutrinos," http://arxiv.org/abs/ 1109.5172. In press.

[24] G. Amelino-Camelia, "Phenomenology of philosophy of science: OPERA data," http://arxiv.org/abs/1206.3554. In press.

[25] G. Y. Bogoslovsky, "Dynamic rearrangement of vacuum and the phase transitions in the geometric structure of space-time," http://arxiv.org/abs/1201.2521. In press.

[26] D. Faroughy, Finite Special Relativity and Definite Upper Limits For the Velocities, Masses and the Energies of Massive Particles, San Jose State University, SJSU, San Jose, Calif, USA, 1985.

[27] Inspire/Spires Records and D. Faroughy, Mass \& Quantum Dependent Chiral Lorentz Transformations and a New Theory of Ether, University of Lavern, Lavern, Calif, USA, 1988.

[28] D. Faroughy, "Several action and action deformation proposals in $f(R)$ variable $G$ modified gravities and a brief expose on variable G ontology," http://arxiv.org/abs/0712.0450. In press.

[29] S. R. Coleman and S. L. Glashow, "High-energy tests of Lorentz invariance," Physical Review D, vol. 59, no. 11, p. 116008, 1999.

[30] A. G. Cohen and S. L. Glashow, "New constraints on neutrino velocities," http://arxiv.org/abs/1109.6562. In press.
[31] F. Bezrukov and H. M. Lee, "Model dependence of the bremsstrahlung effects from the superluminal neutrino at OPERA," http://arxiv.org/abs/1112.1299. In press.

[32] U. D. Jentschura and B. J. Wundt, "An infinitesimally superluminal neutrino is left-handed, conserves Lepton number and solves the autobahn paradox (Illustrative Discussion)," http://arxiv.org/abs/1206.6342. In press.

[33] P. Abreu, M. Aglietta, E.J. Ahn et al., "Update on the correlation of the highest energy cosmic rays with nearby extragalactic matter," Astroparticle Physics, vol. 34, no. 5, pp. 314-326, 2010.

[34] A. V. Glushkov, "On the anisotropy of $E_{0} \geq 5.5 \mathrm{eV}$ cosmic rays according to data of the Pierre Auger collaboration," http://arxiv.org/abs/1202.4520. In press.

[35] S. A. Thomas, F. B. Abdalla, and O. Lahav, "Upper bound of $0.28 \mathrm{eV}$ on neutrino masses from the largest photometric Redshift survey," Physical Review Letters, vol. 105, no. 3, p. 031301, 2010.

[36] The Pierre Auger Collaboration, P. Abreu, M. Aglietta, and M. Ahlers, "A search for ultra-high energy neutrinos in highly inclined events at the Pierre Auger Observatory," http://arxiv .org/abs/1202.1493. In press.

[37] M. Dresden and A. Albano, "Nonlinear space-time transformations related to the Lorentz group," Proceedings of the National Academy of Sciences of the United States of America, vol. 58, no. 3, pp. 916-922, 1967.

[38] A. Lyra and M. Carvalho, "A geometrical setting for constructing nonlinear Lorentz transformations of the AlbanoDresden type," Physics Research International, vol. 2011, Article ID 302164, 9 pages, 2011. 

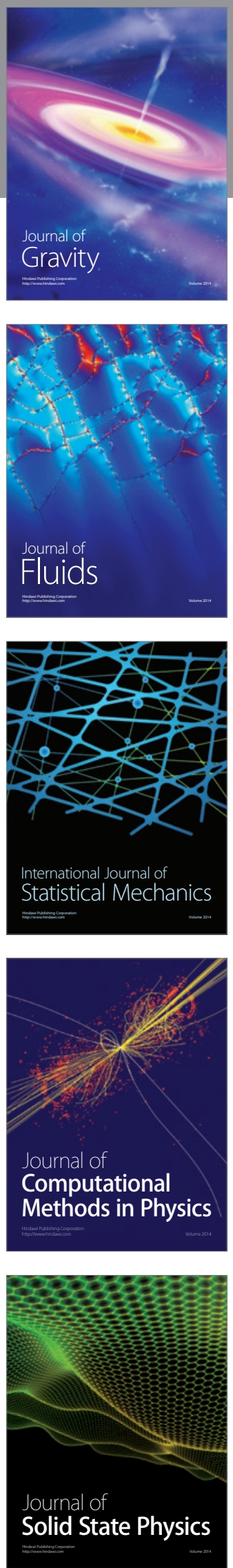

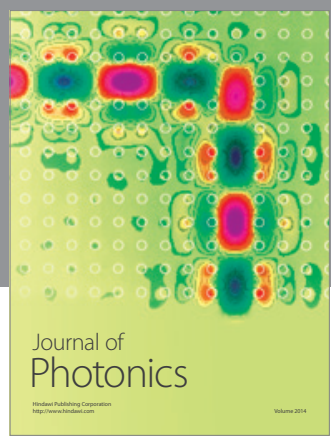

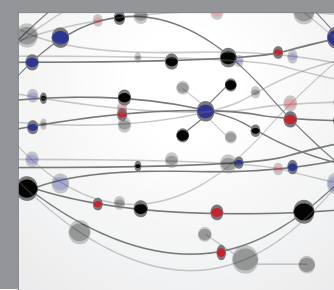

The Scientific World Journal

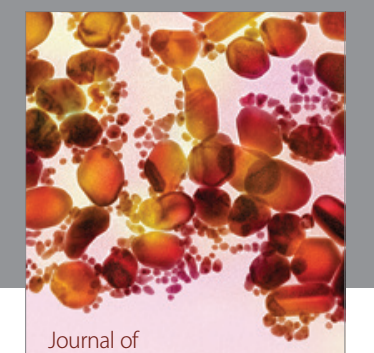

Soft Matter
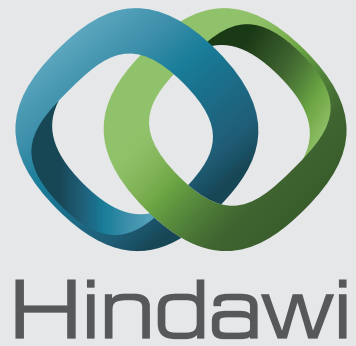

Submit your manuscripts at

http://www.hindawi.com
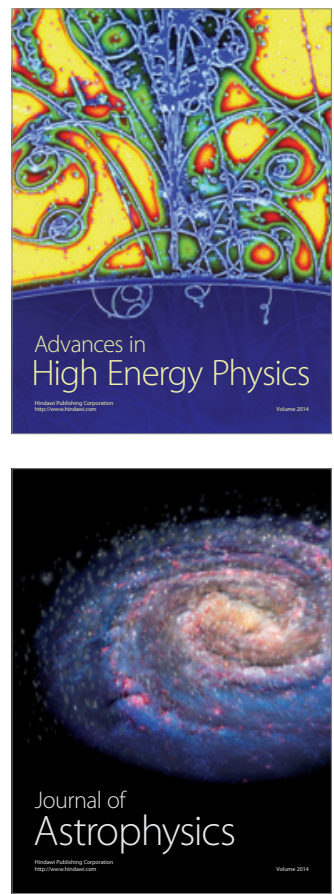
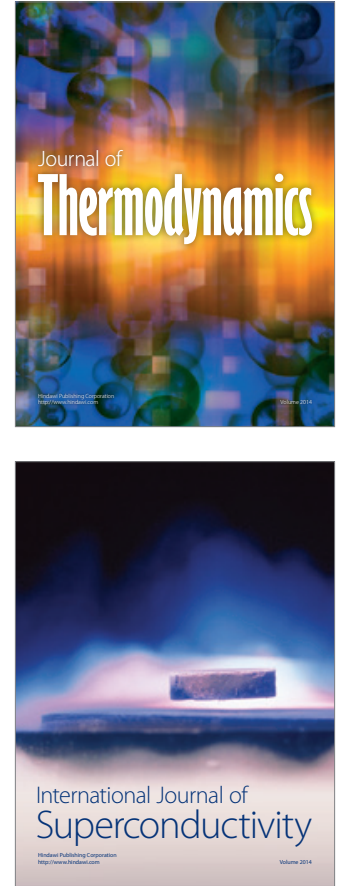
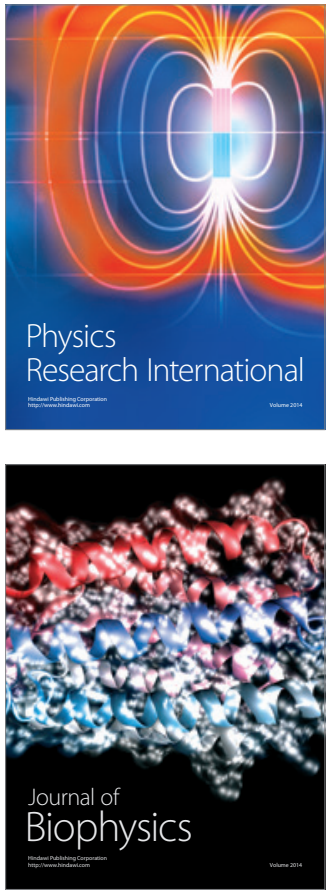
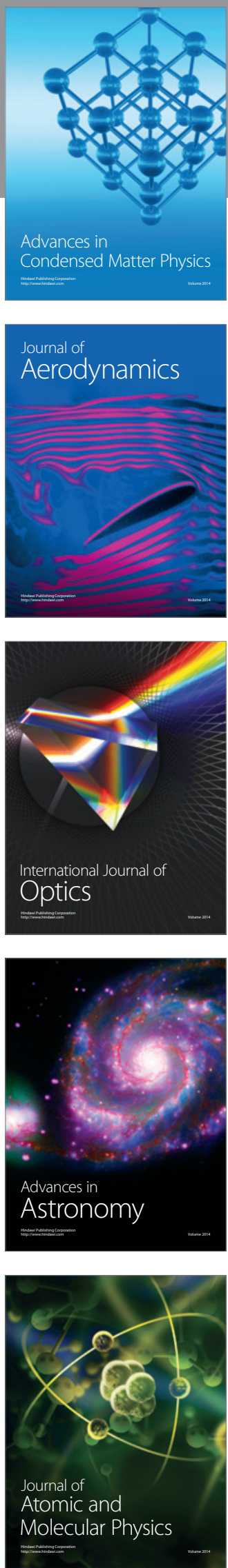